\title{
Electromagnetic effects on trace impurity transport in tokamak plasmas
}

\author{
T. Hein and C. Angioni \\ Max-Planck-Institut für Plasmaphysik, IPP-EURATOM Association, D-85748 Garching bei \\ München, Germany
}

(March 12, 2010)

The impact of electromagnetic effects on the transport of light and heavy impurities in tokamak plasmas is investigated by means of an extensive set of linear gyrokinetic numerical calculations with the code GYRO (J. Candy and R. E. Waltz, J. Comput. Phys. 186, 545 (2003)) and of analytical derivations with a fluid model. The impurity transport is studied by appropriately separating diffusive and convective contributions, and conditions of background microturbulence dominated by both ion temperature gradient (ITG) and trapped electron modes (TEM) are analyzed. The dominant contribution from magnetic flutter transport turns out to be of pure convective type. However it remains small, below $10 \%$, with respect to the $E \times B$ transport. A significant impact on the impurity transport due to an increase of the plasma normalized pressure parameter $\beta$ is observed in the case of ITG modes, while for TEM the overall effect remains weak. In realistic conditions of high $\beta$ plasmas in the high confinement $\left(\mathrm{H}_{-}^{-}\right)$mode, with dominant ITG turbulence, the impurity diffusivity is found to decrease with increasing $\beta$ in qualitative agreement with recent observations in tokamaks. In contrast, in these conditions, the ratio of the total off-diagonal convective velocity to the diagonal diffusivity is not strongly affected by an increase of $\beta$, particularly at low impurity charge, due to a compensation between the different off-diagonal contributions. 
(Some figures in this article are in color only in the electronic version)

52.25.Fi, 52.30.Gz, 52.35.Qz, 52.55.Fa

\section{INTRODUCTION}

While the presence of impurities in the edge and divertor regions of a tokamak has beneficial effects like the reduction of heat loads on the first wall elements and a cold gas target around the divertor, in the central region it might imply serious limitations of performance and operation in a fusion reactor. Thus, the possibility of reaching plasma conditions with reduced inward convection or even outward convection of impurities in the core is extremely attractive for reactor operation. This is of particular importance in the presence of highly charged plasma facing components, like W.

In this framework, the increasing interest in high $\beta_{N}$ scenarios, like the hybrid scenario [1-3] for ITER [4] operation, motivates the study of the impact of electromagnetic effects at finite $\beta$ on the transport of impurities from the theoretical standpoint. Here $\beta_{N}=$ $\beta /\left(I_{P} / a B_{T}\right)$ with $\beta=\langle p\rangle /\left(B_{T}^{2} / 8 \pi\right)$ where $\langle p\rangle$ is the volume average kinetic pressure, $I_{p}$ the plasma current, $a$ the plasma minor radius and $B_{T}$ the toroidal magnetic field.

The impact of finite $\beta$ effects on light impurity transport has received some consideration from the experimental side $[5,6]$. A decrease of the He diffusivity with increasing $\beta$ has been observed in DIII-D [5], and, consistently, a decrease of trace T diffusivity with increasing $\beta$ has been documented in the Joint European Torus (JET) [6]. The consistency between these experimental observations provides an important test for theory validation in the prediction of the effect of increasing $\beta$ on the transport of impurities. This is of particular relevance for nuclear fusion applications, since a decrease of He diffusivity with increasing $\beta$ might have negative consequences on the central He ash concentration due to the effect of the central He ash particle source in a burning plasma. We consider this problem in the present paper, and, by this, we aim at complementing recent theoretical works dedicated to impurity turbulent transport in the electrostatic limit [7-17]. 
Both light and heavy impurities are considered. We show that for experimentally achievable values of $\beta$, electromagnetic effects can be significant in some conditions. By means of an approach already applied in electrostatic calculations [12], we identify diffusive and convective contributions to the impurity transport, and compute both the $E \times B$ as well as the magnetic flutter [18] transport. The decomposition of the fluxes in diffusive and convective contributions allows the direct comparison with the experimental results. Experimental measurements, usually obtained by means of the analysis of transients produced by gas puff or laser ablation of impurities, yield separately the values of the diffusion coefficient and of the total convective velocity.

In order to reach the purpose of our investigations, and to consider a large range of impurity charges in different turbulence conditions, an extensive set of numerical calculations is required exploring different domains of plasma parameters. For this reason, we felt obliged to limit our study to the calculation of quasi-linear transport, while nonlinear electromagnetic simulations of impurity transport, which are highly demanding in computer time, are left for future work. The quasi-linear impurity transport is calculated using the gyrokinetic simulation code GYRO [19,20]. Plasma parameters providing both ion temperature gradient (ITG) and trapped electron modes (TEM) as unstable microinstabilities in the background have been considered. Analytical derivations, starting from a simple fluid model, are performed in order to shed light on the physics processes which are responsible of the numerical gyrokinetic results.

The paper is organized as follows. The next section introduces the ITG and TEM reference cases and shows the numerical results, investigating the effects of $\beta$ on impurity transport as a function of the impurity charge $\left(Z_{p}\right)$ and mass $\left(A_{p}\right)$. Section III presents the simple fluid model, and the analytical calculation of the electromagnetic effects on impurity transport, used for the interpretation of the numerical results. In section IV, the specific case of typical realistic parameters of an $\mathrm{H}$-mode plasma at mid-radius are considered. The results are compared qualitatively with the experimental observations documented in the literature and discussed in projection to the operation of future fusion devices like ITER. 
Finally, the last section draws the main conclusions of the work.

\section{GYROKINETIC CALCULATIONS OF ELECTROMAGNETIC EFFECTS ON TRACE IMPURITY TRANSPORT}

In this section, results of gyrokinetic calculations are reported, in which a third particle species, namely an impurity, is included in very small charge concentration. The gyrokinetic equation for a particle species $j$ is linear in the normalized logarithmic temperature and density gradients, respectively $R / L_{T j}=-R \nabla_{r} T_{j} / T_{j}$ and $R / L_{n j}=-R \nabla_{r} n_{j} / n_{j}$, where $\mathrm{R}$ is the major radius of the tokamak. Therefore, in a local description, a formal expression for the linear response of the perturbed distribution function $\delta f_{j}$ to an electromagnetic potential fluctuation

$$
U=\delta \Phi-v_{\|} / c \delta A_{\|}
$$

where $v_{\|}, \delta \phi$ and $\delta A_{\|}$are parallel velocity, electrostatic and parallel vector potential fluctuations, respectively, is provided by the following linear combination of these gradients,

$$
\delta f_{j}=\left(A_{j} \frac{R}{L_{n j}}+B_{j} \frac{R}{L_{T j}}+C_{j}\right) U
$$

where the coefficients $A_{j}, B_{j}$ and $C_{j}$ do not depend on the logarithmic gradients themselves, but are complicated functions in the phase space.

The particle flux $\Gamma_{j}$ in the radial direction is given by

$$
\Gamma_{j}=\left\langle\left.\int d^{3} v \delta f_{j}\left(\mathbf{B} \times \nabla U / B^{2}\right)\right|_{r}\right\rangle,
$$

where the symbol \langle\rangle describes the flux surface average. As a direct consequence, the linearity in the gradients is formally preserved, leading to an expression for the particle flux which is usually written as

$$
\frac{R \Gamma_{j}}{n_{j}}=D_{j} \frac{R}{L_{n j}}+D_{T j} \frac{R}{L_{T j}}+R V_{P j}
$$


where $D_{j}$ denotes the diagonal diffusion coefficient, $D_{T j}$ the thermodiffusion coefficient and $V_{P j}$ is the pure convection velocity. While such an expresson provides a useful physical decomposition, it cannot be considered as a real linear relationship for bulk plasma species, since the transport coefficients depend on the logarithmic gradients. However, for an impurity species $p$ with a charge concentration $n_{p} Z_{p} / n_{e}$ small enough to be negligible in the Poisson and Ampère equations, the coefficients $D_{p}, D_{T p}$ and $V_{P p}$ become independent of the logarithmic density and temperature gradients of that species and the impurity behaves like a trace. The flux of this trace species is a linear function of the logarithmic gradients. In particular, $V_{P p}$ provides the residual flux in the absence of gradients of the impurity, that is when $R / L_{n p}=0$ and $R / L_{T p}=0$.

As a consequence of such a linear relationship, the three coefficients $D_{j}, D_{T j}$ and $V_{P j}$ can be easily computed with a gyrokinetic code using an appropriate set of linear gyrokinetic simulations. Recasting Eq. (4) in the form,

$$
\frac{R \Gamma_{p}}{n_{p}}=D_{p}\left(\frac{R}{L_{n p}}+C_{T} \frac{R}{L_{T p}}+C_{P}\right),
$$

the coefficients $C_{T}=D_{T p} / D_{p}$ and $C_{P}=R V_{P p} / D_{p}$ can be identified from the slope of the particle flux as a function of the logarithmic temperature and density gradients, as well as from the residual flux at zero gradients. In stationary conditions, in the absence of a source and for negligible neoclassical transport, the turbulent flux is at the null, namely $\Gamma_{p}=0$, and the coefficients $C_{T}$ and $C_{P}$ provide the stationary logarithmic density gradient, through the relationship $R / L_{n p}=-C_{T} R / L_{T p}-C_{P}$. It is worth mentioning that first comparisons between linear and nonlinear gyrokinetic calculations of these parameters in the electrostatic limit have shown a satisfactorily good agreement [16].

Here, such an approach is applied in order to compute the electromagnetic effects on the transport of an impurity. The gyrokinetic code GYRO is used for linear flux tube simulations. We include an impurity species with a charge concentration of $1 / 1000$ in a plasma of deuterons and electrons. In order to investigate the different role of ion temperature gradient (ITG) and trapped electron mode (TEM) microinstabilities, the fol- 
lowing reference cases have been considered. The ITG case is defined by the gradients $R / L_{T i}=9$ and $R / L_{T e}=R / L_{n i}=R / L_{n e}=3$, while for the TEM case $R / L_{T e}=9$ and $R / L_{T i}=R / L_{n i}=R / L_{n e}=3$. Common parameters are the safety factor $q=1.4$, the magnetic shear $s=r / q \partial q / \partial r=0.8$ and the local inverse aspect ratio $\epsilon=r_{\text {loc }} / R=0.17\left(r_{\text {loc }}\right.$ is the local minor radius). A s- $\alpha$ geometry [21] is assumed, and ion, impurity and electron temperatures have been chosen to be equal. Collisions are excluded.

The linear dependences of the impurity flux on the logarithmic gradients have been verified in both linear and nonlinear electrostatic simulations [8,12,16]. Fig. 1 shows for the ITG case that a linear dependence is also given in calculations at a finite $\beta_{e}$ of $1 \%$ for both the $E \times B(\mathrm{a}, \mathrm{c})$ and the magnetic flutter component from fluctuations of $A_{\|}[18,22,23](\mathrm{b}, \mathrm{d})$ of the flux as functions of $R / L_{n p}$ and $R / L_{T p}$, respectively, where $\beta_{e}$ takes electromagnetic effects into account. Here, a single value of $k_{y} \rho_{s}=0.3$ in the spectrum has been selected, where $k_{y}$ is the binormal (almost poloidal) wave number and $\rho_{s}=\sqrt{T_{e} m_{i}} / e B$ is the Larmor radius computed with the sound speed. The contributions are calculated consistently in such a way that the sums of the $E \times B$ and the magnetic flutter components give the total flux. The dimensionless transport coefficients $C_{T}$ and $C_{P}$ become independent of the saturation amplitude of the fluctuating electrostatic and magnetic potentials, which makes them particularly suited for linear calculations.

Such a normalization cannot be applied directly to the dimensional impurity diffusion coefficient. However, an appropriate and experimentally relevant normalization can be easily identified. We define the total effective heat conductivity $\chi_{\text {eff }}$ of the plasma such that

$$
\frac{Q_{t o t}}{n T}=\frac{Q_{i}}{n T}+\frac{Q_{e}}{n T}=\chi_{\mathrm{eff}} \frac{\nabla T}{T},
$$

where $Q_{t o t}, Q_{i}$ and $Q_{e}$ are the total, ion and electron heat fluxes, respectively, $T=\left(T_{i}+T_{e}\right) / 2$ and $\nabla T / T=\nabla\left(T_{i}+T_{e}\right) /\left(T_{i}+T_{e}\right)$. Using the definitions in Eq. (6), it can be directly derived that

$$
\chi_{\mathrm{eff}}=\frac{2\left(\chi_{i} \cdot \nabla T_{i} / T_{i} \cdot T_{i} / T+\chi_{e} \cdot \nabla T_{e} / T_{e} \cdot T_{e} / T\right)}{\nabla T_{i} / T_{i} \cdot T_{i} / T+\nabla T_{e} / T_{e} \cdot T_{e} / T}
$$


and therefore with $T_{i}=T_{e}$ and $\nabla T_{i}=\nabla T_{e}, \chi_{\mathrm{eff}}=\chi_{i}+\chi_{e}$, where $\chi_{i}$ and $\chi_{e}$ are the ion and electron effective heat conductivities, respectively. These can be computed within the quasilinear gyrokinetic model by $\chi_{i, e}(r)=-Q_{i, e}(r) / n_{i, e} \partial T_{i, e} / \partial r$ with

$$
Q_{i, e}(r)=\left\langle\left.\int d^{3} v \frac{m_{i, e} v^{2}}{2} \delta f_{i, e}\left(\mathbf{B} \times \nabla U / B^{2}\right)\right|_{r}\right\rangle .
$$

These coefficients correspond to the power balance heat conductivities determined in experiments, which easily allows direct comparisons between our quasi-linear results and the experimental observations.

We separate $E \times B$ and magnetic flutter transport by the following definitions

$$
C_{T}=\left(C_{T}\right)_{E \times B}+\left(C_{T}\right)_{F l}=\frac{D_{E}}{D_{t o t}} C_{T E}+\frac{D_{F}}{D_{t o t}} C_{T F}
$$

and

$$
C_{P}=\left(C_{P}\right)_{E \times B}+\left(C_{P}\right)_{F l}=\frac{D_{E}}{D_{t o t}} C_{P E}+\frac{D_{F}}{D_{t o t}} C_{P F}
$$

where $D_{E}$ and $D_{F}$ are the diagonal diffusion coefficients with $D_{t o t}=D_{E}+D_{F}, C_{T E}=$ $D_{T E} / D_{E}, C_{T F}=D_{T F} / D_{F}, C_{P E}=R V_{P E} / D_{E}$ and $C_{P F}=R V_{P F} / D_{F}$. The quantities $D_{T E}$ and $D_{T F}$ are the thermodiffusion coefficients and $V_{P E}$ and $V_{P F}$ are the pure convective velocities due to $E \times B(\mathrm{E})$ and magnetic flutter $(\mathrm{F})$ transport, respectively.

Figure 2 shows the poloidal spectrum of the linear eigenfrequency $\hat{\omega}_{r}=\omega_{r} / \omega_{D i}$ (a) and growth rate $\hat{\gamma}=\gamma / \omega_{D i}(\mathrm{~b})$, as well as the $E \times B$ and magnetic flutter (Fl) components of the transport coefficients $D_{p, E \times B} / \chi_{\text {eff }}(\mathrm{c}+\mathrm{d}),\left(C_{T}\right)_{E \times B}(\mathrm{e}+\mathrm{f})$ and $\left(C_{P}\right)_{E \times B}(\mathrm{~g}+\mathrm{h})$ for the ITG and TEM cases, with helium as impurity species. We normalize all frequencies to the ion fluid curvature and $\nabla B$ drift frequency $\omega_{D i}=2 k_{y} \rho_{i} v_{i} / R$, with $v_{i}^{2}=T_{i} / m_{i}$ and $\rho_{i}=\sqrt{T_{i} m_{i}} / e B$. The value of $\beta_{e}=0.5 \%$ has been chosen. The spectrum of $\hat{\gamma}$ shows its maximum at $k_{y} \rho_{s} \approx 0.3$ in the ITG case, whereas for TEM a continuous increase up to high poloidal wave numbers is found, since also electron temperature gradient (ETG) modes are unstable for this case, because we have chosen a reference case with $T_{e}=T_{i}$.

The magnetic flutter transport reveals a significant dependence on the poloidal wave number, and is strongly reduced at large values of $k_{y} \rho_{s}$. Therefore, we expect significant 
differences for this part of the transport in nonlinear simulations. However, it remains small with respect to the total transport, with a contribution which is of the order of $1 \%$ (or $10 \%$ for $C_{P}$ ) of the $E \times B$ transport. The latter, instead, does not exhibit strong variations as a function of the poloidal wave number. For this reason, for the qualitative purposes of the present study and considering also the large number of calculations required to explore the dependences of impurity species with various charge and mass in different turbulence conditions, we limited ourselves to perform all the calculations presented in the followings at the single representative value of $k_{y} \rho_{s}=0.3$, where the maximum growth-rate in the ITG case occurs. We mention that quasi-linear calculations to be applied for more quantitative comparisons with experiments should involve the computation of the entire long wave length spectrum, with the inclusion of a model for the spectral dependence of the fluctuation amplitude of the potentials, as made for instance in [11].

In figures $3 \mathrm{a}$ and $3 \mathrm{~b}$ the electromagnetic dependence of the normalized eigenfrequency and linear growth rate are shown for helium as impurity species. The values of $\hat{\omega}_{r}$, being positive for modes in the ion diamagnetic direction and negative in the electron diamagnetic direction, remain almost unchanged with increasing $\beta_{e}$ in both cases. In contrast, the linear growth rate $\hat{\gamma}$ shows different behaviors in the ITG and TEM cases, it decreases with increasing $\beta$ in the ITG reference case, while it remains almost unaffected in the TEM case $[24,25]$. We shall show in the next section, that this difference in the behavior of the growth rate is responsible for the differences found in the the dependence of impurity transport on $\beta$ in the ITG and TEM cases.

Figure 3 shows also the behavior of the coefficients $D_{p} / \chi_{\mathrm{eff}}(\mathrm{c}, \mathrm{d}), C_{T}(\mathrm{e}, \mathrm{f})$ and $C_{P}(\mathrm{~g}, \mathrm{~h})$ as a function of $\beta_{e}$ in linear gyrokinetic simulations, both for $E \times B$ and magnetic flutter (Fl) transport, respectively. First, transport due to $E \times B$ motion plays the major role. It is interesting to note that the dominant contribution of the magnetic flutter transport has the nature of a pure convection and can be up to $10 \%$ in the ITG case at high $\beta_{e}$. On the contrary, the diagonal diffusion and thermodiffusion contributions provided by magnetic flutter are $\approx 1 \%$ in the ITG case, or even smaller for the TEM case. Second, $D_{p, E \times B} / \chi_{\text {eff }}$ decreases 
with $\beta_{e}$ in the ITG case, while in the TEM case it is slightly increasing. As we shall show, the reduction of $D_{p, E \times B} / \chi_{\text {eff }}$ in the ITG case is connected with the reduction of $\hat{\gamma}$, where $D_{p, E \times B}$ decreases stronger that $\chi_{\text {eff }}$. As usual, in both cases positive values of $D_{p, E \times B} / \chi_{\text {eff }}$ are obtained, that is outward diagonal diffusion. This is in contrast to the magnetic flutter component. Moreover, for all the flutter components of the transport coefficients, the directions exhibit a change going from ITG to TEM. Third, both the thermodiffusion and the pure convection are directed inwards in the TEM case, as indicated by the negative value of the coefficients $\left(C_{T}\right)_{E \times B}$ and $\left(C_{P}\right)_{E \times B}$. For ITG modes, the thermodiffusion is directed outwards. The present results agree with previous results in the electrostatic limit $[26,27,8,10,11]$. In the case of TEM, electromagnetic effects are weak, while they play a strong role in the ITG case. In particular, the increase of the thermodiffusion factor $\left(C_{T}\right)_{E \times B}$ in the outward direction can dominate over the increase of the pure convection term $\left(C_{P}\right)_{E \times B}$ in the inward direction (we remind, that in the calculation of the total impurity flux $\Gamma_{p}$ the coefficient $\left(C_{T}\right)_{E \times B}$ has to be multiplied by $R / L_{T p}$, which is $\geq 5$ for typical parameters at mid-radius). These sorts of behavior can be compared to claculations using a spectrum up to a maximum value of 0.7 in $k_{y} \rho_{s}$. The transport coefficients have been calculated by weighting each contribution according to $\gamma /\left\langle k_{\perp}^{2}\right\rangle$ as it has been suggested in [28], and are indicated as open symbols in Figure 3. While for the $E \times B$ contribution in the ITG reference case we do not find large differences, which is consistent with comparisons of linear and nonlinear results in [16], the ratio $D_{p} / \chi_{\text {eff }}$ is slightly reduced in the TEM case, and $\left(C_{P}\right)_{E \times B}$ shows a small decrease with increasing $\beta_{e}$. As already mentioned before, the flutter coefficients show a stronger contribution on the poloidal wave number. Its contribution to the transport coefficients is increased as compared with the calculations at a single $k_{y} \rho_{s}=0.3$ by the fact that $\gamma /\left\langle k_{\perp}^{2}\right\rangle$ peaks around 0.12 (cp. Figure 2).

At this point, it is of interest to investigate the behavior of trace transport coefficients due to changes in the impurity charge $\left(Z_{p}\right)$ and mass $\left(A_{p}\right)$. Figure 4 shows the coefficients $D_{p} / \chi_{\text {eff }}(\mathrm{a}-\mathrm{d}), C_{T}(\mathrm{e}-\mathrm{h})$ and $C_{P}$ (i-l) for the ITG (left panel) and TEM (right panel) cases with increasing $Z_{p}=A_{p} / 2$. Contributions from $E \times B$ and magnetic flutter (Fl) have been 
separated, and we observe again that $E \times B$ transport exceeds the latter by at least a factor of 10 in the ITG case and more than a factor 100 in the TEM case for small $Z_{p}=A_{p} / 2$. With increasing impurity charge, the flutter contribution becomes completely negligible for diagonal diffusion and thermodiffusion at all $\beta_{e}$, while it reaches a finite asymptotic value for pure convection at large $Z_{p}$. The charge dependence of $D_{p, E \times B} / \chi_{\text {eff }}$ for different impurity species is not strongly affected by electromagnetic effects because all the curves at different charge are almost parallel. In contrast, $\left(C_{T}\right)_{E \times B}$ and $\left(C_{P}\right)_{E \times B}$ exhibit different dependences on $\beta$ depending on the impurity charge, in particular, dependences are stronger for light impurities.

In order to separate the effects of impurity charge and mass on the transport coefficients, also a scan of $Z_{p}$ at fixed $A_{p}=1$ has been performed. They are presented in Fig. 5. For $D_{p, E \times B} / \chi_{\text {eff }}$ the behavior for both ITG and TEM is recovered. Therefore, the impurity charge is the quantity dominating diagonal diffusion coefficients. The mass $A_{p}$ weakly lowers the values of $D_{p, E \times B} / \chi_{\text {eff }}$, see Figs. $4 \mathrm{c}$ and $5 \mathrm{c}$ (for instance for $Z_{p}=1$ ). The coefficients $D_{p} / \chi_{\text {eff }}$ and $C_{T}$ show an asymptotic behavior with increasing $Z_{p}$, while $C_{P}$ grows linearly at high values of $Z_{p}$, as expected from previous results [8]. Compared to Fig. 4, this shows that the impurity mass counteracts the effect of charge at finite values of $\beta_{e}$ for both $E \times B$ and flutter diagonal and thermodiffusion, and prevents the linear increase of the pure convection. Obviously, the relative magnitude between $E \times B$ and magnetic flutter transport cannot be considered realistic for these cases with fixed mass.

In the next section, a simplified fluid model is used in order to shed light on the main physical processes which are responsible of the dependence of the impurity transport on $\beta_{e}$ and of the results found in the numerical calculations.

\section{FLUID MODEL}

In order to understand the physics mechanisms which are responsible of the numerical results for the transport coefficients $D_{p}, C_{T}$ and $C_{P}$ including electromagnetic effects, a fluid 
model is considered following Refs. [29-33,8]. The model is kept simple enough in order to allow us to compute analytically the main electromagnetic effects on impurity transport. An important element in the following derivations is the relationship between the fluctuating electrostatic potential $\delta \phi$ and the magnetic field perturbation $\delta A_{\|}$, which is provided by the dynamics of passing electrons.

\section{A. Electromagnetic fluid model}

We summarize the main equations derived in previous works ( $[29-33,8])$. These are essential to understand the origin of the analytical expressions of the transport coefficients, which will be given later. The continuity equation for the trace impurity species is given by

$$
\frac{\partial n_{p}}{\partial t}+\nabla\left(n_{p} \mathbf{v}_{E}\right)+\nabla\left(n_{p} \mathbf{v}_{* p}\right)+\nabla\left(n_{p} \hat{\mathbf{e}}_{||} v_{\| p}\right)=0
$$

where the second term includes the $\mathrm{E} \times \mathrm{B}$ velocity, the third the diamagnetic drift, and the last the parallel velocity. Drift contributions due to polarization and off-diagonal elements of the stress tensor are neglected, since they appear as finite Larmor radius corrections. Using harmonic perturbations of the form $n_{p}=n_{p, 0}+\tilde{n}_{p}=n_{p, 0}+\delta n_{p} \exp (i \mathbf{k} \cdot \mathbf{x}-i \omega t)$ and normalizing all frequencies to the ion fluid curvature and $\nabla B$ drift frequency $\omega_{D i}$, Eq. (11) can be written in the form,

$$
-\hat{\omega} \hat{n}_{p}+\frac{\hat{n}_{p}+\hat{T}_{p}}{Z_{p}}-\left(\frac{R}{2 L_{n p}}-1\right) \frac{T_{e}}{T_{p}} \hat{\phi}+\hat{k}_{\|} \hat{v}_{\| p}=0 .
$$

Here, $\hat{n}_{p}=\delta n_{p} / n_{p, 0}, \hat{T}_{p}=\delta T_{p} / T_{p, 0}$ and $\hat{v}_{\| p}=\delta v_{\|} / v_{i}$ are the normalized density, temperature and parallel velocity fluctuations, $\hat{\phi}=e \delta \phi / T_{e}$ is the normalized fluctuating electrostatic potential, and $\hat{k}_{||}=k_{\|} v_{i} / \omega_{D i}$ is the normalized wave vector parallel to the magnetic field (note that the system has been assumed in the absence of an equilibrium parallel velocity). The second term arises from a finite value of the divergence of the diamagnetic drift, which is in fact a curvature effect (and therefore proportional to $1 / Z_{p}$ ), the third term includes $\mathrm{E} \times \mathrm{B}$ advection and compression independent of charge, and the last term describes compression in parallel velocity. 
The equation for the parallel velocity fluctuations is given by

$$
m_{p} n_{p} \frac{\partial v_{\| p}}{\partial t}=-Z_{p} e n_{p}\left[\nabla_{\|} \phi+\frac{1}{c}\left(\frac{\partial A_{\|}}{\partial t}-\left(\mathbf{v}_{* p} \times\left(\nabla \times A_{\|} \hat{e}_{\|}\right)\right) \hat{e}_{\|}\right)\right]-\nabla_{\|}\left(n_{p} T_{p}\right),
$$

where the second and the third terms on the right hand side take electromagnetic effects into account via the (fluctuating) magnetic vector potential $A_{\|}$[31]. Linearizing Eq. (13) in the fluctuating quantities, and including the same normalizations, the parallel force balance reads

$$
-\frac{A_{p}}{A_{i}} \hat{\omega} \hat{v}_{\| p}+\frac{Z_{p} T_{e}}{T_{p}} \hat{k}_{\| p} \hat{\phi}-\frac{Z_{p} T_{e}}{T_{p}} \frac{v_{i}}{c}\left[\hat{\omega}-\frac{1}{Z_{p}}\left(\frac{R}{2 L_{n p}}+\frac{R}{2 L_{T p}}\right)\right] \hat{A}_{\|}+\hat{k}_{\| \mid}\left(\hat{n}_{p}+\hat{T}_{p}\right)=0,
$$

where we introduced $\hat{A}_{\|}=e \delta A_{\|} / T_{e}$ and assumed $T_{i}=T_{p}$ and $Z_{i}=1$ without loss of generality. The factor in front of $\hat{A}_{\|}$is composed of terms proportional to the logarithmic gradients of impurity density and temperature profiles, arising from the diamagnetic drift velocity, and a term proportional to the complex eigenfrequency arising from the time derivative $\partial_{t} A_{\|}$. This indicates that the inclusion of electromagnetic effects yields additional terms in the impurity transport proportional to $R / L_{n p}$, namely of diffusive type, proportional to $R / L_{T p}$, namely of thermodiffusive type, and of pure convective type, being not proportional to impurity gradients. This is consistent with the results of the numerical gyrokinetic calculations presented in the previous section.

The energy balance equation reads

$$
\frac{3}{2} n_{p}\left(\frac{\partial}{\partial t}+\mathbf{v}_{p} \nabla\right) T_{p}+n_{p} T_{p} \nabla \mathbf{v}_{p}=-\nabla q_{p}
$$

where $\mathbf{v}_{p}$ again includes the fluid drifts. An analogous derivation as for the previous equations, neglecting finite Larmor radius effects, leads to

$$
\left(-\hat{\omega}+\frac{5}{3 Z_{p}}\right) \hat{T}_{p}+\frac{2}{3} \hat{\omega} \hat{n}_{p}-\left(\frac{R}{2 L_{T p}}-\frac{R}{3 L_{n p}}\right) \frac{T_{e}}{T_{p}} \hat{\phi}=0 .
$$

The physical meaning of each term is analogous to that described in Eq. (12). The fluid closure is chosen in such a way that the total heat flux $q_{p}$ is equal to the diamagnetic heat flow $q_{*}$, while parallel heat flows are explicitly neglected [29]. Note that the parallel fluid motion is not included in Eq. (16) since its contribution drops in the linearization. Moreover, collisions are excluded. 


\section{B. Relationship between fluctuating potentials $\hat{\phi}$ and $\hat{A}_{\|}$}

Electromagnetic effects can enter the fluid equations of the trace impurity mainly in two ways. The first is through changes in the eigenfrequency $\hat{\omega}$ of the background microinstability due to an increase of $\beta$, which affects already the electrostatic part of the impurity fluid equations. The second is through additional terms proportional to $\hat{A}_{\|}$in the impurity parallel motion equation. The gyrokinetic results of the previous sections have shown that in most cases radial transport due to $E \times B$ motion exceeds magnetic flutter transport by two orders of magnitude. Therefore, we concentrate on the $E \times B$ transport, first. We shall discuss briefly some aspects of the magnetic flutter part at the end of this Section. The main impact of changes of $\hat{\omega}$ due to an increase of $\beta_{e}$ can be studied calculating the phase shift between $\hat{n}_{p}$ and $\hat{\phi}$ using the impurity fluid equations in the electrostatic limit. Instead the calculations of additional direct terms depending on $\beta$ in the impurity fluid equations involve the calculation of the relationship between $\hat{A}_{\|}$and $\hat{\phi}$, provided by passing electron dynamics. This is briefly presented in this subsection.

Following [31,33], and combining passing electron continuity equation and parallel force balance with the Ampère's law, the relationship between the fluctuating electrostatic $\hat{\phi}$ and magnetic vector potential $\hat{A}_{\|}$can be derived. Temperature fluctuations of passing electrons are assumed in the isothermal limit, where inertia is neglected. The result expressed with the normalizations introduced in the previous subsection reads,

$$
\hat{A}_{\|}=\hat{k}_{\|} \frac{c}{v_{i}} \frac{-T_{e} / T_{i} R / 2 L_{n e}-\hat{\omega}}{\hat{\omega}\left(T_{e} / T_{i} R / 2 L_{n e}-\hat{\omega}\right)-T_{e} / T_{i}\left[\hat{\omega}-\left(1+\eta_{e}\right) T_{e} / T_{i} R / 2 L_{n e}\right]+k_{\perp}^{2} \rho_{i}^{2} k_{\|}^{2} v_{A}^{2} / v_{i}^{2}} \hat{\phi}
$$

In a more compact form, this can be written as

$$
\hat{A}_{\|}=\hat{k}_{||} \hat{\Omega}(\hat{\omega}) \hat{\phi}
$$

The Alfvén velocity is given by $v_{A}^{2}=B^{2} /\left(4 \pi n_{e} m_{i}\right)$. In order to identify the explicit occurrence of electromagnetic terms in Eq. (17), a re-arrangement of the third term in the denominator is made. It will be shown, that this one is the leading contribution in the 
limit of small $\beta_{e}$. Assuming $n_{e}=n_{i}$, one finds $v_{A}^{2} / v_{i}^{2}=2 / \beta_{i}$ with $\beta_{i}=T_{e} / T_{i} \beta_{e}$. The definition of $k_{\|}=(q R)^{-1}$ in our simple model leads to the normalized parallel wave vector $\hat{k}_{\|}^{2}=\left(4 q^{2} k_{y}^{2} \rho_{i}^{2}\right)^{-1}$. Therefore the last term is $k_{\perp}^{2} \rho_{i}^{2} k_{\|}^{2} v_{A}^{2} / v_{i}^{2}=k_{\perp}^{2} /\left(2 q^{2} k_{y}^{2} \beta_{i}\right)$. Since usually $\hat{\omega}=\mathcal{O}(1)$ and, in the limit of small $\beta_{i}, k_{\perp}^{2} /\left(2 q^{2} k_{y}^{2} \beta_{i}\right)=\mathcal{O}(10)$ to $\mathcal{O}(100)$ (assuming $k_{\perp} \approx k_{y}$ ), we identify the leading terms in $\hat{\Omega}(\hat{\omega})$, Eq. (18) via

$$
\hat{\Omega}(\hat{\omega})=-2 \frac{c}{v_{i}} \beta_{i} q^{2} \frac{k_{\perp}^{2}}{k_{y}^{2}}\left(\frac{T_{e}}{T_{i}} \frac{R}{2 L_{n e}}+\hat{\omega}\right) .
$$

To summarize, the fluid model consisting of Eqs. (12), (14), (16) and (17) (or with (19) for small- $\beta_{i}$ approximation) allows an analytical derivation of the trace impurity particle transport coefficients $D_{p}, C_{T}$ and $C_{P}$. This will be completed in the next section.

\section{Analytical $E \times B$ transport coefficients}

We study the impact of electromagnetic effects on the $E \times B$ transport. As already pointed out in the last section, electromagnetic effects can occur in two ways. The first way is through the modification of the phase shift between impurity density fluctuations and electrostatic potential fluctuations in the electrostatic limit produced by changes of the eigenfrequencies in response to an increase of $\beta$ in the background plasma. The second way is by means of additional terms in the impurity flux introduced directly by fluctuations in the parallel vector potential. We find that the first way is that which produces the dominant effects, and therefore we start by discussing this one. We focus first on the ratio of the impurity diffusivity to the total effective heat conductivity $D_{p, E \times B} / \chi_{\text {eff }}$. The effect of changing $\beta_{e}$ on impurity diagonal diffusivity and total effective heat conductivity is calculated adopting the fluid model consisting of the continuity equation, the energy balance equation and the parallel motion equation of the trace species. As just mentioned, we neglect the explicit $\beta_{e}$ dependence, i.e. the electromagnetic contribution to the Lorentz force, but we concentrate on the effect of $\hat{\gamma}$ in the electrostatic expression. According to Fig. 3b, the linear growth rate remains almost constant with increasing $\beta_{e}$ for the TEM case while it strongly 
decreases in the ITG case. Thus, in the latter case, where $\chi_{\text {eff }}$ is mainly determined by the ion heat conductivity, the ratio $D_{p, E \times B} / \chi_{\text {eff }}$ due to this model is given by the coefficients

$$
\begin{aligned}
D_{p, E \times B}= & 2 \frac{\gamma \hat{\gamma}^{2}}{k_{x}^{2}}\left\{|\hat{\omega}|^{2}\left(\frac{1}{2}|\hat{\omega}|^{2}-\frac{7}{3 Z_{p}} \hat{\omega}_{r}+\frac{55}{18 Z_{p}^{2}}\right)\right. \\
& \left.+\hat{k}_{\|}^{2} \frac{A_{i}}{A_{p}}\left[\frac{1}{6}\left(7 \hat{\gamma}^{2}-\hat{\omega}_{r}^{2}\right)+\frac{5}{9}\left(\hat{k}_{\|}^{2} \frac{A_{i}}{A_{p}}+\frac{\hat{\omega}_{r}}{Z_{p}}\right)+\frac{25}{18 Z_{p}^{2}}\right]\right\} . \\
& \left\{\frac { 1 } { 9 A _ { i } ^ { 2 } Z _ { p } ^ { 4 } } \left\{A_{p}^{2}|\hat{\omega}|^{2}\left[9 Z_{p}^{4}\left(|\hat{\omega}|^{2}\right)^{2}+10 Z_{p}^{2}\left(13 \hat{\omega}_{r}^{2}+7 \hat{\gamma}^{2}\right)+25-100 Z_{p} \hat{\omega}_{r}-60 Z_{p}^{3} \hat{\omega}_{r}|\hat{\omega}|^{2}\right]+\right.\right. \\
& 10 \hat{k}_{\|}^{2} A_{i} A_{p}\left[5 Z_{p} \hat{\omega}_{r}-15 Z_{p}^{2} \hat{\omega}_{r}^{2}+13 Z_{p}^{3} \hat{\omega}_{r}^{3}-3 Z_{p}^{4} \hat{\omega}_{r}^{4}+3 Z_{p}^{4} \hat{\gamma}^{4}+Z_{p}^{2} \hat{\gamma}^{2}\left(5+Z_{p} \hat{\omega}_{r}\right)\right]+ \\
& \left.\left.25 \hat{k}_{\|}^{4} A_{p}^{2} Z_{p}^{2}\left[Z_{p}^{2} \hat{\gamma}^{2}+\left(Z_{p} \hat{\omega}_{r}-1\right)^{2}\right]\right\}\right\}^{-1}
\end{aligned}
$$

and

$$
\begin{aligned}
\chi_{i}= & 2 \frac{\gamma \hat{\gamma}^{2}}{k_{x}^{2}}\left\{|\hat{\omega}|^{2}\left(\frac{1}{2}|\hat{\omega}|^{2}-\hat{\omega}_{r}+\frac{5}{6}\right)+\frac{\hat{k}_{\|}^{2}}{3}\left(4 \hat{\gamma}^{2}-2 \hat{\omega}_{r}^{2}+5 \hat{\omega}_{r}\right)+\frac{5}{6} \hat{k}_{\|}^{4}+\right. \\
& \frac{L_{T i}}{L_{n i}}\left[|\hat{\omega}|^{2}\left(\frac{2}{3} \hat{\omega}_{r}-\frac{10}{9}\right)+\frac{\hat{k}_{\|}^{2}}{3}\left(3 \hat{\omega}_{r}^{2}-\hat{\gamma}^{2}-\frac{20}{3} \hat{\omega}_{r}\right)-\frac{5}{9} \hat{k}_{\|\|}^{4}\right]+ \\
& \left.\frac{L_{T i}}{R}\left[|\hat{\omega}|^{2}\left(-\frac{2}{3}|\hat{\omega}|^{2}-\frac{4}{3} \hat{k}_{\|}^{2} \hat{\omega}_{r}+\frac{10}{9}\left(1+\hat{k}_{\|}^{2}\right)\right)+\frac{20}{9} \hat{k}_{\| \mid}^{2} \hat{\omega}_{r}+\frac{10}{9} \hat{k}_{\|\|}^{4}\right]\right\} \\
& \left\{\frac { 1 } { 9 } \left\{|\hat{\omega}|^{2}\left[9\left(|\hat{\omega}|^{2}\right)^{2}+10\left(13 \hat{\omega}_{r}^{2}+7 \hat{\gamma}^{2}\right)+25-100 \hat{\omega}_{r}-60 \hat{\omega}_{r}|\hat{\omega}|^{2}\right]+\right.\right. \\
& 10 \hat{k}_{\| \mid}^{2}\left[5 \hat{\omega}_{r}-15 \hat{\omega}_{r}^{2}+13 \hat{\omega}_{r}^{3}-3 \hat{\omega}_{r}^{4}+3 \hat{\gamma}^{4}+\hat{\gamma}^{2}\left(5+\hat{\omega}_{r}\right)\right]+ \\
& \left.\left.25 \hat{k}_{\| \mid}^{4}\left[\hat{\gamma}^{2}+\left(\hat{\omega}_{r}-1\right)^{2}\right]\right\}\right\}^{-1},
\end{aligned}
$$

where we expanded $\hat{\omega}=\hat{\omega}_{r}+i \hat{\gamma}$ in the fluid equations such that $|\hat{\omega}|^{2}=\hat{\omega}_{r}^{2}+\hat{\gamma}^{2}$. The usual mixing length estimate applied in Ref. [29] has been used, where the saturation level is assumed to be provided by the balance between linear growth rate and the dominant $E \times B$ convective nonlinearity.

In order to explain the behavior of the ratio $D_{p, E \times B} / \chi_{\mathrm{eff}}$, it turns out that parallel motion, which has been included in the lengthy calculation of Eq. (20) and Eq. (21), is important, because in this way the diagonal diffusion coefficient decreases in the ITG case with increasing $\beta_{e}$ due to its strong dependence on $\hat{\gamma}$, while $\chi_{\text {eff }}$ only weakly decreases with increasing $\beta_{e}$. This leads to a ratio $D_{p, E \times B} / \chi_{\text {eff }}$, which decreases with decreasing 
$\hat{\gamma}$, in agreement with the gyrokinetic results. For impurities with high $Z_{p}=A_{p} / 2$, the decrease of $D_{p, E \times B}$ with raising $\beta_{e}$ becomes stronger, but also the ratio of the respective denominators increases. This implies that the decrease of $D_{p, E \times B} / \chi_{\text {eff }}$ is not strongly affected by increasing impurity charge, as also found in the gyrokinetic results, Figs. 4 and 5 . The ratio $D_{p, E \times B} / \chi_{\text {eff }}$ is mainly determined by the dependence of $D_{p, E \times B}$ with increasing $\beta_{e}$. We underline that, like often performed in experimental comparisons between particle diffusion and heat conduction, the coefficient $D_{p, E \times B}$ is the diagonal impurity diffusivity, while $\chi_{\mathrm{eff}}$ is the total power balance (effective) heat conductivity. Therefore, only a part of the phase shift between $\hat{n}$ and $\hat{\phi}$ is considered in the former case, while in the latter one the total phase shift between $\hat{T}$ and $\hat{\phi}$ is taken into account. In the TEM case, the linear growth rate is changing very weakly with increasing $\beta_{e}$, and therefore neither $D_{p, E \times B}$ nor $\chi_{\text {eff }}$ show a significant change, which explains why $D_{p, E \times B} / \chi_{\text {eff }}$ remains almost constant in gyrokinetic simulations.

Now, we turn to the effect caused by the variation of linear growth rates with increasing $\beta$ on the coefficients $C_{T}$ and $C_{P}$ produced by electrostatic fluctuations. This can be readily discussed using the expressions in [8]. We recall that, neglecting parallel velocity fluctuations, the electrostatic $E \times B$ thermodiffusion coefficient for the impurities reads,

$$
\left(C_{T}\right)_{E S, T}=\frac{2}{Z_{p}} \frac{\hat{\omega}_{r}-5 /\left(3 Z_{p}\right)}{|\hat{\omega}|^{2}-14 \hat{\omega}_{r} /\left(3 Z_{p}\right)+55 /\left(9 Z_{p}^{2}\right)},
$$

where again $|\hat{\omega}|^{2}=\hat{\omega}_{r}^{2}+\hat{\gamma}^{2}$. We observe therefore that a decrease of $\hat{\gamma}$ with increasing $\beta$ leads to a decrease of the denominator and therefore an increase of the coefficient $C_{T}$, as found in the gyrokinetic results for the ITG reference case. On the contrary, in the TEM case the linear growth rates are only weakly affected with consequent small changes of the impurity thermodiffusive coefficient.

The same considerations can be made for the pure convection coefficient $C_{P}$. Neglecting again parallel velocity fluctuations, $\left(C_{P}\right)_{E S, T}=-2\left(1+2\left(C_{T}\right)_{E S, T} / 3\right)$, which consistently provides an increase in size of $C_{P}$ in the inward direction due to an increase of $C_{T}$ in the case of the ITG mode, while it remains almost constant in the TEM case. The consistency with 
the gyrokinetic results indicates that the main mechanism through which an increase of $\beta$ affects the transport of impurities derives from the coupling between density and temperature fluctuations produced by the $\nabla B$ and curvature drift.

It is certainly of interest for the physical understanding to investigate also the dependence of thermodiffusion and pure convection on the impurity charge and mass by means of the fluid model. In addition, this allows us to more directly separate transport processes due to perpendicular drifts from those arizing from parallel dynamics. To this end, we consider the full electrostatic fluid model, including also the electrostatic part of the parallel motion equation, and we note that in the limit of very large impurity charge, that is $Z_{p}>>1$, the coupling between density fluctuations and parallel velocity fluctuations is the only one which does not vanish. In the thermodiffusion coefficient, parallel dynamics introduces additional terms with respect to Eq. (22), yielding the following more complete expression

$$
\begin{aligned}
\left(C_{T}\right)_{E S}= & \left\{\frac{|\hat{\omega}|^{2}}{Z_{p}}\left(\hat{\omega}_{r}-\frac{5}{3 Z_{p}}\right)+\frac{1}{2} \hat{k}_{\|}^{2} \frac{A_{i}}{A_{p}}\left(3 \hat{\omega}_{r}^{2}-\hat{\gamma}^{2}-\frac{5}{3} \hat{k}_{\|}^{2} \frac{A_{i}}{A_{p}}\right)-\frac{10}{3 Z_{p}} \hat{k}_{\|}^{2} \frac{A_{i}}{A_{p}} \hat{\omega}_{r}\right\} . \\
& \left\{|\hat{\omega}|^{2}\left(\frac{1}{2}|\hat{\omega}|^{2}-\frac{7}{3 Z_{p}} \hat{\omega}_{r}+\frac{55}{18 Z_{p}^{2}}\right)\right. \\
& \left.+\hat{k}_{\|}^{2} \frac{A_{i}}{A_{p}}\left[\frac{1}{6}\left(7 \hat{\gamma}^{2}-\hat{\omega}_{r}^{2}\right)+\frac{5}{9}\left(\hat{k}_{\|}^{2} \frac{A_{i}}{A_{p}}+\frac{\hat{\omega}_{r}}{Z_{p}}\right)+\frac{25}{18 Z_{p}^{2}}\right]\right\}^{-1},
\end{aligned}
$$

which reduces to Eq. (22) in the limit of $\hat{k}_{\|}^{2}=0$. From this expression, it is evident that at high $Z_{p}$ and high mass $A_{p}=2 Z_{p}$ the thermodiffusion coefficient vanishes. The role of parallel dynamics can be put in evidence by studying the behavior in the ideal limit of large $Z_{p}$ with fixed impurity mass $A_{p}=1$, as we have already made in the numerical gyrokinetic results presented in Fig. 5. We observe that with $Z_{p}>>1$ and $A_{p}=1$ the thermodiffusion coefficient $\left(C_{T}\right)_{E S}$ does not vanish in the limit $\beta_{e}=0$ for both ITG and TEM instabilities, which means positive or negative values of $\hat{\omega}_{r}$, but decreases to finite small values. In addition, in the ITG case, the coefficient $\left(C_{T}\right)_{E S}$ is found to increase in the limit $Z_{p}>>1$ with increasing $\beta_{e}$ and therefore with decreasing of $\hat{\gamma}$, due to the concurrent increase of the numerator and the decrease of the denominator, consistently with the gyrokinetic results shown in Fig. 5. In contrast, no strong dependence on $\beta_{e}$ is obtained in the TEM case, 
where the eigenfrequencies are found to very weakly depend on $\beta_{e}$.

We turn now to the effect of parallel dynamics on the pure convection coefficient. Neglecting temperature fluctuations this is directly given by the results in [8],

$$
\left(C_{P}\right)_{E S \|}=-\frac{2|\hat{\omega}|^{2}+4 \hat{\omega}_{r} \hat{k}_{\|}^{2} Z_{p} A_{i} / A_{p}}{|\hat{\omega}|^{2}+\hat{k}_{\|}^{2} A_{i} / A_{p}} .
$$

This yields an inward contribution, increasing in size with increasing $Z_{p}$ for the ITG case (positive $\hat{\omega}_{r}$ ). In contrast, in the TEM case it is directed inward for small impurity charge, but reverses direction with increasing charge due to the outward contribution of the second term at the numerator (negative $\hat{\omega}_{r}$ ). These behaviors are also found in the numerical gyrokinetic results presented in Fig. 4, in the limit of $\beta_{e}=0$. The dependence on $\beta_{e}$ found in the ITG case can be also explained by the simple fluid model, observing that with increasing $\beta_{e}$ and therefore decreasing $\hat{\gamma}$, the second term $-4 \hat{\omega}_{r} \hat{k}_{\|}^{2} Z_{p} A_{i} / A_{p} /\left(|\hat{\omega}|^{2}+\hat{k}_{\|}^{2} A_{i} / A_{p}\right)$ in Eq. (24) increases in size, in the inward direction.

All these considerations based on the simple fluid model, and the consistency found with the more complete numerical gyrokinetic results, allow us to explain the dependences on $\beta_{e}$ and impurity charge and mass found in the gyrokinetic calculations and to identify the main physical processes which are responsible for these. The main dependences arize from electrostatic type of transport processes, mainly connected with the perpendicular dynamics. An increase of $\beta_{e}$ affects the electrostatic impurity transport through the dependence on $\beta_{e}$ of the eigenfrequencies, and in particular the growth rate, of the background instabilities.

However, as we were mentioning above, in addition to the influence of the variation of the linear growth rates on the electrostatic part of the impurity flux, electromagnetic effects on the $E \times B$ transport are produced also by additional contributions due to the presence of electromagnetic fluctuations in $A_{\|}$, which imply additional terms proportional to $\beta$ itself. Despite the fact that these play only a minor role in the relatively low $\beta$ limit of typical tokamak plasmas, we find interesting to explore their specific dependences on the mode frequency with the analytical model. In order to single out the electromagnetic contribution proportional to $\beta$, and adopt equations simple enough to perform analytical calculations, 
we keep only the electromagnetic part in the parallel motion equation,

$$
\hat{v}_{\| p}=\frac{A_{i}}{A_{p}} \cdot \frac{1}{\hat{\omega}}\left\{Z_{p} \frac{T_{e}}{T_{p}} \frac{v_{i}}{c}\left[\frac{1}{Z_{p}}\left(\frac{R}{2 L_{n p}}+\frac{R}{2 L_{T p}}\right)-\hat{\omega}\right]\right\} \hat{A}_{\| \cdot}
$$

In addition, temperature fluctuations, i.e. the $\hat{T}_{p}$ term in the continuity equation, whose main effect has been considered in the previous discussion, are neglected, and, by this, the coupling with the energy balance is removed, which reduces the order of the algebraic system. As already underlined, the coupling between continuity and parallel motion equation is actually the only one remaining in the limit of large $Z_{p}$.

Including the relationship between the fluctuating potentials $\hat{\phi}$ and $\hat{A}_{\|}$given by Eq. (17), one finds

$$
\hat{n}_{p}=\left(-\hat{\omega}^{2}+\frac{\hat{\omega}}{Z_{p}}\right)^{-1} \cdot\left\{\left(\frac{R}{2 L_{n p}}-1\right) \frac{T_{e}}{T_{p}} \hat{\omega}+\hat{K}\left[\hat{\omega}-\frac{1}{Z_{p}}\left(\frac{R}{2 L_{n p}}+\frac{R}{2 L_{T p}}\right)\right] \hat{\phi}\right\},
$$

where

$$
\hat{K}=\hat{k}_{\|}^{2} Z_{p} \frac{A_{i}}{A_{p}} \frac{T_{e}}{T_{p}} \frac{v_{i}}{c} \hat{\Omega}(\hat{\omega})
$$

By expanding the complex quantities as follows, $\hat{\omega}=\hat{\omega}_{r}+i \hat{\gamma}, \hat{K}=\hat{K}_{r}+i \hat{K}_{i}$, the results for the transport coefficients are

$$
\begin{gathered}
D_{p, E \times B}=\frac{\gamma}{k_{x}^{2}} \frac{\hat{\gamma}}{|\hat{N}|^{2}}\left\{\hat{\gamma}|\hat{\omega}|^{2}+\frac{T_{p}}{Z_{p} T_{e}}\left[\hat{K}_{r}\left(\frac{\hat{\gamma}}{Z_{p}}-2 \hat{\gamma} \hat{\omega}_{r}\right)-\hat{K}_{i}\left(\hat{\gamma}^{2}+\frac{\hat{\omega}_{r}}{Z_{p}}-\hat{\omega}_{r}^{2}\right)\right]\right\}, \\
D_{p, E \times B} \cdot\left(C_{T}\right)_{E \times B}=\frac{\gamma}{k_{x}^{2}} \frac{\hat{\gamma}}{|\hat{N}|^{2}} \frac{T_{p}}{Z_{p} T_{e}}\left\{\hat{K}_{r}\left(\frac{\hat{\gamma}}{Z_{p}}-2 \hat{\gamma} \hat{\omega}_{r}\right)-\hat{K}_{i}\left(\hat{\gamma}^{2}+\frac{\hat{\omega}_{r}}{Z_{p}}-\hat{\omega}_{r}^{2}\right)\right\}, \\
D_{p, E \times B} \cdot\left(C_{P}\right)_{E \times B}=\frac{2 \gamma}{k_{x}^{2}} \frac{\hat{\gamma}}{|\hat{N}|^{2}} \frac{T_{p}}{T_{e}}|\hat{\omega}|^{2}\left\{-\frac{T_{e}}{T_{p}} \hat{\gamma}+\hat{K}_{r} \hat{\gamma}+\hat{K}_{i}\left(\frac{1}{Z_{p}}-\hat{\omega}_{r}\right)\right\}, \\
|\hat{N}|^{2}=|\hat{\omega}|^{2}\left(|\hat{\omega}|^{2}-\frac{2 \hat{\omega}_{r}}{Z_{p}}+\frac{1}{Z_{p}^{2}}\right) .
\end{gathered}
$$

The terms proportional to $\hat{K}_{r}$ or $\hat{K}_{i}$ identify explicitly electromagnetic contributions through their dependence on $\beta_{i}$. The first term in $D_{p, E \times B}$ is the usual term due to $E \times B$ advection, 
while the second and the third include the additional term due to $\hat{A}_{\|}$proportional to the density gradient in the Lorentz force, which has its origin in the diamagnetic drift. Within these, the contributions proportional to $\left(1 / Z_{p}\right)$ enter due to the divergence of the diamagnetic heat flow, which is in fact a curvature effect. In $D_{p, E \times B}\left(C_{T}\right)_{E \times B}$ the same terms as the last two in $D_{p, E \times B}$ appear due to the additional term proportional to the temperature gradient. The electrostatic part in $D_{p, E \times B}\left(C_{P}\right)_{E \times B}$ occurs from $E \times B$ compression, and the last two arize again from electromagnetic additions proportional to $\hat{\omega}$ in the Lorentz force (coming from the $\partial_{t} A_{\|}$term), where contributions proportional to $\left(1 / Z_{p}\right)$ are again due to curvature effects.

In order to simplify these results, $D_{p, E \times B}$ as well as the dimensionless quantities $\left(C_{T}\right)_{E \times B}$ and $\left(C_{P}\right)_{E \times B}$ are calculated in the limit of small $\beta_{i}$, i.e. using Eq. (19). After this reduction, $\hat{K}_{r}$ includes a term $\propto R / L_{n e}$ arising from the $E \times B$ advection term in the electron continuity equation used to relate $\hat{A}_{\|}$to $\hat{\phi}$, and a term $\propto \hat{\omega}_{r}$, while $\hat{K}_{i}$ is proportional to $\hat{\gamma}$ only. The latter two are due to the time derivative of the fluctuating electron density. Then, the additional contributions proportional to $\beta$ in the transport coefficients are,

$$
\begin{gathered}
D_{p, E \times B}=\frac{\gamma}{k_{x}^{2}} \frac{\hat{\gamma}^{2}}{|\hat{N}|^{2}}\left\{\frac{1}{2} \frac{A_{i}}{A_{p}} \frac{\beta_{i}}{k_{\perp}^{2} \rho_{i}^{2}}\left[\frac{T_{e}}{T_{p}} \frac{R}{2 L_{n e}}\left(2 \hat{\omega}_{r}-\frac{1}{Z_{p}}\right)+|\hat{\omega}|^{2}\right]\right\}, \\
\left(C_{T}\right)_{E \times B}=\frac{1}{2} \frac{A_{i}}{A_{p}} \frac{\beta_{i}}{k_{\perp}^{2} \rho_{i}^{2}}\left[\frac{1}{|\hat{\omega}|^{2}} \frac{T_{e}}{T_{i}} \frac{R}{2 L_{n e}}\left(2 \hat{\omega}_{r}-\frac{1}{Z_{p}}\right)+1\right],
\end{gathered}
$$

and

$$
\left(C_{P}\right)_{E \times B}=-Z_{p} \frac{A_{i}}{A_{p}} \frac{\beta_{i}}{k_{\perp}^{2} \rho_{i}^{2}}\left(\frac{T_{e}}{T_{i}} \frac{R}{2 L_{n e}}+\frac{1}{Z_{p}}\right) .
$$

Here, only the leading order electromagnetic terms $\mathcal{O}\left(\beta_{i}^{1}\right)$ have been taken into account. The first and second terms in $D_{p, E \times B}$ and $\left(C_{T}\right)_{E \times B}$ have the same origin, namely $E \times B$ advection and time derivative of the fluctuating electron density, respectively. The coefficient $\left(C_{P}\right)_{E \times B}$ is given by $E \times B$ advection and a curvature term. Eqn. (32), (33) and (34) provide small additions on the charge and mass dependence of trace impurity coefficients $D_{p, E \times B},\left(C_{T}\right)_{E \times B}$ and $\left(C_{P}\right)_{E \times B}$ in electromagnetic simulations. 
We observe that these contributions proportional to $\beta$ are such that in the case of diffusion and thermodiffusion the coefficients $D_{p, E \times B}$ and $\left(C_{T}\right)_{E \times B}$ depend on the real frequency of the mode, and consist of terms which are all in the outward direction for the case of a ITG mode $\left(\hat{\omega}_{r}>0\right)$, while they can balance in the case of TEM $\left(\hat{\omega}_{r}<0\right)$. In the thermodiffusion coefficient, this part proportional to $\beta$ enhances the effect previously described in the electrostatic part of the flux by the reduction of the growth rate of the instability with increasing $\beta$. On the contrary, the part of the coefficient $\left(C_{P}\right)_{E \times B}$ proportional to $\beta$ given by Eq. (34), increases in size in the inward direction with increasing $\beta_{e}$ for both the ITG and the TEM case.

\section{Analytical magnetic flutter transport coefficients}

Although the magnetic flutter contribution to the total transport is small in the low $\beta_{e}$ limit, the gyrokinetic results exhibit the interesting feature that magnetic flutter transport components can reverse their direction from the ITG to the TEM instabilities. This is particularly the case for the dominant term of the magnetic flutter transport, that is the pure convective flux. In order to investigate the main physical reason behind the numerical results, we applied the same fluid model also to the analytical calculation of the magnetic flutter flux $\Gamma_{F l} / n_{p}=-k_{y}\left\langle\tilde{v}_{\| p} A_{\|} / B\right\rangle$, and we compare it with the gyrokinetic results. The complete analytical calculation of the complex phase relationship between parallel velocity fluctuations $\tilde{v}_{\| p}$ and parallel magnetic vector potential fluctuations $\hat{A}_{\|}$is as lengthy as that for the $E \times B$ transport, which instead implies the calculation of the complex phase relationship between density and electrostatic potential fluctuations. However, a rather strong simplification can be obtained keeping only first order terms in $\beta$ in the expression for the flux and focusing on the calculation of the term in the pure convective contribution which survives in the limit of highly charged impurities. This shall allow us to provide an example of the dependence of the direction of the magnetic flutter transport on the sign of the real frequency of the unstable mode. First, we note that, since $\hat{A}_{\|} \propto \beta_{e}$, terms 
directly proportional to $\hat{A}_{\|}$in the expression of $\tilde{v}_{\| p}$ given by Eq. (14), which stem from the electromagnetic part of the Lorentz force in the parallel motion equation, yield contributions which are proportional to $\beta^{2}$ in the final expression of the flux and therefore can be neglected. Moreover, we note that in Eq. (14), the effect of the parallel pressure gradient term vanishes with increasing impurity inertia, while the parallel gradient of the electrostatic potential fluctuations produces a residual effect at high charge, due to the presence of the charge in the Lorentz force. Of course, through the parallel pressure gradient, contributions to the parallel velocty fluctuations which are proportional to the radial logarithmic gradients of the equilibrium impurity density and temperature $R / L_{n p}$ and $R / L_{T p}$ occur, and these will not be included in our analytical calculation. By focuysing only on the leading term for highly charged impurities, our calculation will deliver a pure convective term only. For such a term, the calculation of the phase relationship between $\hat{\phi}$ and $\hat{A}_{\|}$, which is first order in $\beta$, is required. We use Eq. (18), in the small $\beta$ limit. We underline that it depends critically on the eigenfrequency of the instability, which occurs here due to the reactive passing electron response given by the continuity equation in the presence of magnetic fluctuations. The analytical expression of the magnetic flutter pure convection coefficient in this limit is,

$$
\left(C_{P}\right)_{F l}=-\frac{4 \gamma \hat{\gamma}^{2}}{k_{x}^{2}|\hat{N}|^{2} D_{p, t o t}} \beta_{i} \hat{k}_{\|}^{2} \frac{k_{\perp}^{2}}{k_{y}^{2}} \frac{A_{i}}{A_{p}} Z_{p} \frac{T_{e}}{T_{p}}\left|\hat{\omega}+\frac{R}{2 L_{n e}} \frac{T_{e}}{T_{i}}\right|^{2}\left(2 \frac{T_{i}}{T_{e}} \hat{\omega}_{r}+\frac{R}{2 L_{n e}}\right),
$$

with

$$
|\hat{N}|^{2}=|\hat{\omega}|^{2}\left(|\hat{\omega}|^{2}-\frac{30}{9 Z_{p}} \hat{\omega}_{r}+\frac{25}{9 Z_{p}^{2}}\right) \cdot\left[|\hat{\omega}|^{2}+\frac{R}{L_{n e}} \frac{T_{e}}{T_{i}}+\left(\frac{R}{2 L_{n e}} \frac{T_{e}}{T_{i}}\right)^{2}\right]
$$

where the prevailing contribution in $D_{p, t o t}$ is given by the $E \times B$ diffusion and therefore is positive. The saturation level has been estimated as before, applying the relationship between $\hat{A}_{\|}$and $\hat{\phi}$, see Eq. (18) in the small $\beta$ limit. The analytical expression can be compared with the gyrokinetic results. We observe that $\left(C_{P}\right)_{F l}$ in $\mathrm{Eq}(35)$ is negative for ITG modes, that is, it describes an inward directed convection. In addition, it can reverse sign in case of TEM instabilities with sufficiently large absolute values of $\hat{\omega}_{r}$. This behavior agrees with the numerical results of our ITG and TEM reference cases presented in Figs. 
3,4 and 5, where a reversal of the sign of the coefficient is obtained in the two cases. It is also interesting to note that while in the TEM case the dependence of $\left(C_{P}\right)_{F l}$ on $\beta$ is linear, since the eigenfrequencies of the instability are weakly affected by an increase of $\beta$ itself, in the ITG case, the dependence on $\beta$ is stronger, since the decrease of the growth rate of the instability with increasing $\beta$ implies a reduction of the denominator in Eq. (35), which leads to a more parabolic type of dependence.

In a similar way, the occurrence of the mode eigenfrequency in the relationship between $\hat{\phi}$ and $\hat{A}_{\|}$impacts also the behavior of the magnetic flutter components of the diagonal diffusion and the thermodiffusion. The calculation of these terms is however longer, since it requires the derivation of the relationship between parallel velocity fluctuations and electrostatic potential fluctuations occurring through the $E \times B$ advection in the impurity continuity and energy balance equations. As already mentioned, these contributions decrease with increasing impurity mass $A_{p}$.

In conclusion, this section allowed us to identify the main physical mechanisms through which an increase of $\beta$ affects the $E \times B$ transport of impurities. We have shown that the main variations are directly connected with the electrostatic part of the flux, which is modified by to the influence of $\beta$ on the growth rate of the instability in the background plasma. In particular for the off-diagonal coefficients $C_{T}$ and $C_{P}$, this is related to the electrostatic coupling between density and temperature impurity fluctuations produced by the $\nabla B$ and curvature drift, and explains why $\beta$ effects are strongly reduced at large values of the impurity charge and mass. At high impurity charges with a fixed impurity mass, additional terms due to parallel motion lead to finite electromagnetic contributions to the thermodiffusion and pure convection coefficients. We have also investigated specifically the additional contributions to the $E \times B$ transport produced by the coupling with the fluctuations of the magnetic parallel vector potential $A_{\|}$, in the small $\beta$ limit. We have found that in the case of the diffusion and thermodiffusion coefficients, these contributions depend on the sign of the real frequency of the mode, and in particular in the ITG case, can enhance the thermodiffusion in the outward direction, which is a particularly relevant 
result for the transport of light impurities. In the case of the pure convection coefficient, the contribution to the transport produced by $A_{\|}$are directed inward for both ITG and TEM, since they are found to be independent of the direction of propagation of the mode. The transport coefficients of the magnetic flutter contribution are given by the phase relation between parallel velocity fluctuations and the fluctuations in the parallel component of the vector potential. The comparison of the explicitly derived pure convection coefficient with the gyrokinetic results indicates that the main physics mechnism responsible for the reversal of the magnetic flutter convection when moving from the ITG to the TEM reference case is connected with the occurence of the mode eigenfrequency in the relationship between the fluctuating electrostatic and vector potentials, due to the non-adiabatic passing electron response in the presence of magnetic flucutations.

\section{IMPURITY TRANSPORT FOR TYPICAL H-MODE PLASMA PARAMETERS}

In this section, calculations of impurity transport including electromagnetic effects are made for typical $\mathrm{H}$-mode parameters around mid-radius. We underline that the chosen plasma parameters are also very close to those predicted for the ITER standard scenario [34], in the dimensionless form which is applied in the gyrokinetic calculations. The logarithmic gradients at mid-radius are $R / L_{T i}=5.0, R / L_{T e}=5.3$ and $R / L_{n}=2.1$, the safety factor is $q=1.1$, the magnetic shear is $s=0.4$, the ion to electron temperature ratio is $T_{e} / T_{i}=1.05$, while the geometry parameters (elongation of 1.5 and triangularity of 0.1 ) have been used in Miller geometry [35]. Collisions have been included. The local collisionality is $\nu_{e i}\left[c_{s} / a\right]=$ 0.02. The dominant micro-instability in these conditions is a ITG mode for all presented values of $\beta_{e}$. Therefore we do not expect very large differences with respect to the ITG reference case followed in the previous sections. However, we consider here the realistic situation of comparable ion and electron logarithmic temperature gradients, which is more suited for at least qualitative comparisons with the experimental results available in the 
literature on the impact of $\beta$ on the transport of impurities. Of course, for quantitative comparisons, the actual measured experimental profiles should be used as input in the gyrokinetic calculations, and, if possible, nonlinear simulations should be performed.

Linear gyrokinetic simulations for the interesting ion species tritium, helium, carbon, nickel and tungsten with a ionization stage $46+$ typical of $\mathrm{H}$-modes in present devices around mid-radius $\left(T_{e} \approx 3 \mathrm{keV}\right)$ are shown in Fig. 6 . Vertical lines in the figure identify the value of $\beta_{e}=1.8 \%$ at the ITER standard scenario parameters, and which can be also considered typical for usual H-mode operation. Results similar to those presented in Fig. 4 are obtained, which indicates that the reference cases discussed in Section II can be considered to some extent generic. Nevertheless, results of Fig. 6 allow us to draw some specific conclusions which we expect to be directly applicable to typical $\mathrm{H}$-mode plasmas.

Magnetic flutter diffusion and thermodiffusion provide only small contributions for all ion species and at all experimentally relevant values of $\beta_{e}$. In contrast, more significant can be the convective part of the magnetic flutter, particularly for fully ionized heavy impurities. The magnetic flutter convection can be as large as $10 \%$ of the corresponding pure convective $E \times B$ transport.

The ratio of the $E \times B$ diagonal diffusion to the total effective conductivity reaches a finite asymptotic value with increasing charge and decreases with increasing $\beta_{e}$ at all charges. As we discussed in the previous Section, this is caused by the drop of the linear growth rate, which is found to affect the diagonal diffusivity much more than the total effective conductivity. The reason for the higher absolute value of $D_{p, E \times B} / \chi_{\text {eff }}$ compared to the ITG parameter study before is the lower $\chi_{\text {eff }}$ due to the lower ion temperature gradient. The theoretical result of a decrease of the light impurity diffusivity with increasing $\beta$ agrees with experimental observations for He transport in DIII-D [5] and for trace T transport in JET [6]. We underline that since the magnitude of diagonal diffusion of He governs the impact of the central He source produced by fusion reactions on the He ash concentration profile, the reduction of $D_{p, E \times B} / \chi_{\text {eff }}$ with $\beta_{e}$ deserves consideration in the prediction of the He ash profile in high $\beta$ operational scenarios and certainly requires further theoretical 
and experimental investigations. We note also that the diffusion coefficient for He in the electrostatic limit agrees with previous linear and nonlinear calculations [16].

In order to underline the differences to the previous parameter studies, we like to discuss an interesting result provided by the coefficient $\left(C_{T}\right)_{E \times B}$ in Fig. 6c. At moderate values of $\beta_{e}$ tritium thermodiffusion is directed inwards, while all heavier impurities move outwards. This behavior can be understood through Eq. (22), and shows that for sufficiently low charges the inward contribution $-5 /\left(3 Z_{p}\right)$ can prevail over the outward contribution provided by $\hat{\omega}_{r}$ for ITG modes. In the present case, with comparable temperature gradients and not too large value of $\hat{\omega}_{r}$, which is typical of reactor relevant $\mathrm{H}$-mode plasmas, such a transition in the direction of thermodiffusion takes place between the charge $Z_{p}=1$ of hydrogenic species and the charge $Z_{p}=2$ of He. We recall that for TEM instabilities, thermodiffusion is always directed inwards, for any particle charge. For $\left(C_{P}\right)_{E \times B}$, negative values are found for all impurity species, that is an inward pure convection. Its absolute value increases with increasing $Z_{p} / A_{p}$, while a reduction of the charge to mass ratio, like in the case of the not fully ionized W, acts to reduce the contribution. The light impurities tritium and helium show only a small increase in the inward direction with increasing $\beta_{e}$ as compared to the ITG parameter studies in the previous section.

Finally, the total convection to diffusion ratio are presented in Fig. 7. Here the thermodiffusion coefficient $C_{T}$ has been multiplied by the actual logarithmic temperature gradient $R / L_{T i}$ and summed to $C_{P}$ to obtain the ratio of the total off-diagonal flux to the diagonal diffusion. As we already illustrated in Section II, such a ratio is equal to the value of $R / L_{n}$ of the impurity in the absence of a particle source and for negligible neoclassical transport in stationary conditions. The $E \times B$ and magnetic flutter parts of the off-diagonal flux have been separated in Fig. 7a and Fig. 7b, respectively, and divided by the total diagonal diffusivity, sum of the $E \times B$ and the magnetic flutter diffusivities. Consistently with all previous results, the magnetic flutter contribution to the ratio $R V / D$ is small for the range of standard operational scenarios $\left(\beta_{e}=1.6 \%-1.9 \%\right)$. Nevertheless, it can reach $\approx 10 \%$ of the $E \times B$ transport at the projected hybrid operation, with $\beta_{e}=2.3 \%-2.6 \%$. The dominant 
$E \times B$ part is increasing with $\beta$ at small values of the impurity charge, as a consequence of the effect of thermodiffusion. This result is of particular interest for He, which turns out to have the smaller value of $R V / D$. On the contrary, the total convection to diffusion ratio decreases (that is, it increases in size, in the inward direction) for heavy impurities. The effect, however, remains small, and no case of real strong accumulation with increasing $\beta$ is predicted to occur, which is a result favorable for nuclear fusion applications.

\section{CONCLUSIONS}

Electromagnetic effects on the transport of trace impurities in tokamaks have been computed using linear gyrokinetic simulations and analytical calculations based on a simplified fluid model. The $E \times B$ and magnetic flutter components of the transport have been calculated separately, in order to investigate their relative contribution to the total impurity flux. Both components have been decomposed in their diffusive, thermodiffusive and pure convective contributions, making use of the linear dependence of the flux of trace impurities on the density and temperature logarithmic gradients. Reference cases with dominant ITG and TEM modes in the plasma background have been considered, and the dependence of the transport on charge and mass investigated.

In conditions of both ITG and TEM instabilities, the transport produced by magnetic flutter is found to be usually only of the order of $1 \%$ with respect to the $E \times B$ transport. Interestingly, the dominant part of flutter transport is of pure convective type. For ITG modes and at large values of $\beta$ it can be as large as $10 \%$ as compared to the pure convection produced by $E \times B$ transport, providing a non-negligible component of the dependence of the pure convection coefficient on $\beta$. The ITG and TEM reference cases allowed us to show that changes in the background instability can strongly affect the dependence of the impurity transport coefficients on $\beta_{e}$. In the ITG case, the diagonal diffusion normalized to the effective total heat conductivity decreases with increasing $\beta_{e}$, while it remains almost constant in the TEM reference case. Thermodiffusion is found to increase in the outward 
direction with increasing $\beta_{e}$ at all impurity charges in the ITG case. This can lead to a reversal of the thermodiffusive flux from inward to outward for very light impurities with increasing $\beta_{e}$, since, differently from heavy impurities, the thermodiffusive contribution of very light impurities can be directed inward in the electrostatic limit in the ITG case. In the TEM case, thermodiffusion is almost independent on $\beta_{e}$ and directed inwards at all charges. The pure convection term is directed inwards in both cases and increases in the inward direction with increasing $\beta_{e}$. The latter effect is stronger in the ITG case, whereas in the TEM case this behavior is rather weak.

The dependence of the transport on the impurity charge is also of interest. It is found that the diagonal $E \times B$ diffusion decreases in the ITG case while it increases in the TEM case with increasing impurity charge. Impurity mass does not have a strong impact. On the other hand, as it can be easily expected from simple considerations, inertia strongly impact the properties of the magnetic flutter transport. In particular, the diagonal flutter diffusion vanishes for heavy impurities. The same is obtained for both $E \times B$ and magnetic flutter thermodiffusion. Pure convection remains finite at high $Z_{p}=A_{p} / 2$ for both the $E \times B$ and the flutter component.

The numerical results have been interpreted by means of a reduced fluid model, consisting of the continuity, the parallel force balance including the electromagnetic Lorentz force terms and the energy balance equation for the impurity species. Analytical expressions for the various impurity transport coefficients have been derived, which have been used in order to identify the main coupling mechanisms leading to electromagnetic effects on impurity transport. It is found that the main effects obtained in the gyrokinetic calculations are due to the impact on the electrostatic part of the $E \times B$ transport produced by the modification of the linear growth rate of the instability in the background plasma with increasing $\beta_{e}$. The main differences between ITG and TEM therefore are directly a consequence of the fact that an increase of $\beta_{e}$ produces a reduction of the linear growth rate in ITG modes, while it affects very weakly the linear growth rate in TEM modes.

The behaviors of diagonal diffusion, thermodiffusion and pure convection have been inves- 
tigated for a realistic case of typical $\mathrm{H}$-mode plasma parameters, similar to those predicted for ITER. The dominant background microinstability turns out to be an ITG mode. Here, more physics effects like plasma shape and collisions are taken into account compared to the reference cases used for parametric studies. The transport coefficients show mainly the same properties as in the reference ITG case. In particular, the decrease of diagonal diffusion with increasing $\beta_{e}$ found in the reference ITG case is confirmed by these more realistic calculations and it is in qualitative agreement with observations obtained with light impurities in DIII-D [5] and JET [6]. This result certainly requires some consideration, since too strong a reduction of diagonal diffusion of He with increasing $\beta_{e}$ might lead to an accumulation of He ash in the center in high $\beta$ scenarios in a reactor due to an increase of the effect of the central He source produced by fusion reactions. Our theoretical result should motivate further experimental investigations on this specific problem and direct quantitative comparisons between theoretical predictions and experimental measurements, in order to increase our predictive capability of the consequences of high $\beta$ operation on He ash accumulation in a burning plasma.

An additional interesting result is related to the behavior of the total off-diagonal transport, sum of thermodiffusion and pure convection. This is directed inwards for impurities at all charges in the realistic $\mathrm{H}$-mode case. The ratio of the total off-diagonal transport to the diagonal diffusion decreases weakly in size with increasing $\beta_{e}$ for light impurities, while it increases in size with increasing $\beta_{e}$ for heavy impurities. Therefore, in the absence of significant central impurity sources and with small neoclassical transport, an increase of $\beta_{e}$ is predicted to produce a small flattening of light impurity density profiles and a peaking of heavy impurity density profiles.

In conclusion, the work presented here is intended as a first step in the theoretical investigation of trace impurity transport in high $\beta$ operational scenarios in tokamaks. The present linear results have to be verified by means of nonlinear simulations, and validated by further experimental studies. Quantitative comparisons between theoretical predictions and the experimental results have to be considered an important step in the future research. 


\section{ACKNOWLEDGMENTS}

The authors are grateful to J. Candy and R. E. Waltz for providing the gyrokinetic code GYRO, and to A. Bottino and B.D. Scott for helpful discussions.

[1] A. C. C. Sips, G. D. Conway, R. Dux, A. Herrmann, J. Hobirk, O. Gruber, S. Gunter, C. Maggi, M. Manso, M. Maraschek, F. Leuterer, Y.-S. Na, A. Peeters, J. Stober, and R. Wolf, Plasma Phys. Controlled Fusion 44, A151 (2002).

[2] T. Luce, M. Wade, J. Ferron, A. Hyatt, A. Kellman, J. Kinsey, R. L. Haye, C. Lasnier, M. Murakami, P. Politzer, and J. Scoville, Nucl. Fusion 43, 321 (2003).

[3] E. Joffrin, A. Sips, J. Artaud, A. Becoulet, L. Bertalot, R. Budny, P. Buratti, P. Belo, C. Challis, F. Crisanti, M. de Baar, P. de Vries, C. Gormezano, C. Giroud, O. Gruber, G. Huysmans, F. Imbeaux, A. Isayama, X. Litaudon, P. Lomas, D. McDonald, Y. Na, S. Pinches, A. Staebler, T. Tala, A. Tuccillo, K.-D. Zastrow, and J.-E. C. to the Work Programme, Nucl. Fusion 45, $626(2005)$.

[4] M. Shimada, D. J. Campbell, V. Mukhovatov, M. Fujiwara, N. Kirneva, K. Lackner, M. Nagami, V. D. Pustovitov, N. Uckan, J. Wesley, N. Asakura, A. E. Costley, A. J. H. Donné, E. J. Doyle, A. Fasoli, C. Gormezano, Y. Gribov, O. Gruber, T. C. Hender, W. Houlberg, S. Ide, Y. Kamada, A. Leonard, B. Lipschultz, A. Loarte, K. Miyamoto, V. Mukhovatov, T. H. Osborne, A. Polevoi and A. C. C. Sips, Nucl. Fusion 47, S1 (2007).

[5] C. C. Petty, T. C. Luce, D. C. McDonald, J. Mandrekas, M. R. Wade, J. Candy, J. G. Cordey, V. Drozdov, T. E. Evans, J. R. Ferron, R. J. Groebner, A. W. Hyatt, G. L. Jackson, R. J. L. Haye, T. H. Osborne, and R. E. Waltz, Phys. Plasmas 11, 2514 (2004).

[6] K.-D. Zastrow, J. M. Adams, Y. Baranov, P. Belo, L. Bertalot, J. H. Brzozowski, C. D. Challis, S. Conroy, M. de Baar, P. de Vries, P. Dumortier, J. Ferreira, L. Garzotti, T. C. 
Hender, E. Joffrin, V. Kiptily, J. Mailloux, D. C. McDonald, R. Neu, M. O’Mullane, M. F. F. Nave, J. Ongena, S. Popovichev, M. Stamp, J. Stober, D. Stork, I. Voitsekhovitch, M. Valovic, H. Weisen, A. D. Whiteford, A. Zabolotsky, and JET EFDA Contributors, Plasma Phys. Controlled Fusion 46, B255 (2004).

[7] C. Estrada-Mila, J. Candy, and R. E. Waltz, Phys. Plasmas 12, 022305 (2005).

[8] C. Angioni and A. G. Peeters, Phys. Rev. Lett. 96, 095003 (2006).

[9] R. Guirlet, C. Giroud, T. Parisot, M. E. Puiatti, C. Bourdelle, L. Carraro, N. Dubuit, X. Garbet, and P. R. Thomas, Plasma Phys. Controlled Fusion 48, B63 (2006).

[10] N. Dubuit, X. Garbet, T. Parisot, R. Guirlet, and C. Bourdelle, Phys. Plasmas 14, 042301 (2007).

[11] C. Bourdelle, X. Garbet, F. Imbeaux, A. Casati, N. Dubuit, R. Guirlet, and T. Parisot, Phys. Plasmas 14, 112501 (2007).

[12] C. Angioni, R. Dux, E. Fable, A. G. Peeters, and the ASDEX Upgrade Team, Plasma Phys. Controlled Fusion 49, 2027 (2007).

[13] H. Nordman, T. Fülöp, J. Candy, P. Strand, and J. Weiland Phys. Plasmas 14052303 (2007).

[14] V. Naulin, J. J. Rasmussen, C. Angioni, C. Giroud, M. Valisa, M. E. Puiatti, L. Carraro, Turbulent impurity transport, 1st ITER international Summer School, Aix-en-Provence (FR), 16-20 Jul 2007, AIP Conference Proceedings 1013, 191 (2008).

[15] H. Nordman, R. Singh, T. Fülöp, L.-G. Eriksson, R. Dumont, J. Anderson, P. Kaw, P. Strand, M. Tokar, and J. Weiland, Phys. Plasmas 15, 042316 (2008).

[16] C. Angioni, A. Peeters, G. Pereverzev, A. Bottino, J. Candy, R. Dux, E. Fable, T. Hein, and R. Waltz, Nucl. Fusion 49, 055013 (2009).

[17] T. Fülöp and H. Nordman, Phys. Plasmas 16, 032306 (2009). 
[18] J. D. Callen, Phys. Rev. Lett. 39, 1540 (1977).

[19] J. Candy and R. E. Waltz, J. Comput. Phys. 186, 545 (2003).

[20] J. Candy and R. E. Waltz, Phys. Rev. Lett. 91, 045001 (2003).

[21] J. W. Connor, R. J. Hastie, and J. B. Taylor, Phys. Rev. Lett. 40, 396 (1978).

[22] A. B. Rechester and M. N. Rosenbluth, Phys. Rev. Lett. 40, 38 (1978).

[23] B. Scott, Plasma Phys. Controlled Fusion 39, 1635 (1997).

[24] L. Vermare, C. Angioni, A. Bottino, A. G. Peeters, and ASDEX Upgrade Team, J. Phys.: Conf. Ser. 123, 012040 (2008).

[25] J. Candy, Phys. Plasmas 12, 072307 (2005).

[26] M. Frojdh, M. Liljestrom, and H. Nordman, Nucl. Fusion 32, 419 (1992).

[27] X. Garbet, N. Dubuit, E. Asp, Y. Sarazin, C. Bourdelle, P. Ghendrih, and G. T. Hoang, Phys. Plasmas 12, $082511(2005)$.

[28] F. Jenko, T. Dannert and C. Angioni, Plasma Phys. Controlled Fusion 47, B195 (2005).

[29] J. Weiland, A. B. Jarmén, and H. Nordman, Nucl. Fusion 29, 1810 (1989).

[30] H. Nordman, J. Weiland, A. B. Jarmén, Nucl. Fusion 30, 983 (1990).

[31] G. Bateman, A. H. Kritz, J. E. Kinsey, A. J. Redd, and J. Weiland, Phys. Plasmas 5, 1793 (1998).

[32] J. Weiland, Collective Modes in Inhomogeneous Plasma - Kinetics and Advanced Fluid Theory (Institute of Physics (IOP) Publishing, Bristol and Philadelphia, 2000).

[33] A. Eriksson and J. Weiland, Phys. Plasmas 12, 092509 (2005).

[34] G. Pereverzev, C. Angioni, A. Peeters, and O. Zolotukhin, Nucl. Fusion 45, 221 (2005).

[35] R. L. Miller, M. S. Chu, J. M. Greene, Y. R. Lin-Liu, and R. E. Waltz, Phys. Plasmas 5, 973 
(1998). 
Figure Captions

FIG. 1. (Color online) Electromagnetic $\left(\beta_{e}=1 \%\right)$ gyrokinetic calculations of particle fluxes $R \Gamma_{p} / n_{p}$ for trace impurity helium (charge concentration $n_{p} Z_{p} / n_{e}=1 / 1000$ ) as a function of the normalized logarithmic gradients $R / L_{n p}(\mathrm{a}, \mathrm{b})$ and $R / L_{T p}(\mathrm{c}, \mathrm{d})$ using GYRO. The background fluctuations in the electron-deuteron plasma have been chosen to be ITG dominated according to the ITG reference case.

FIG. 2. (Color online) Linear gyrokinetic calculations of normalized eigenfrequencies $\hat{\omega}_{r}=\omega_{r} / \omega_{D i}(\mathrm{a})$, growth rates $\hat{\gamma}=\gamma / \omega_{D i}(\mathrm{~b})$, and of the $E \times B$ coefficients $D_{p, E \times B} / \chi_{\mathrm{eff}}(\mathrm{c})$, $\left(C_{T}\right)_{E \times B}(\mathrm{e})$ and $\left(C_{p}\right)_{E \times B}(\mathrm{~g})$, as well as of the corresponding magnetic flutter (Fl) components $(\mathrm{d}, \mathrm{f}, \mathrm{h})$, as a function of $k_{y} \rho_{s}$ for trace impurity helium at $\beta_{e}=0.5 \%$.

FIG. 3. (Color online) Gyrokinetic calculations of normalized eigenfrequencies $\hat{\omega}_{r}=\omega_{r} / \omega_{D i}$ (a), growth rates $\hat{\gamma}=\gamma / \omega_{D i}(\mathrm{~b})$, and of the $E \times B$ coefficients $D_{p, E \times B} / \chi_{\text {eff }}(\mathrm{c}),\left(C_{T}\right)_{E \times B}$ (e) and $\left(C_{p}\right)_{E \times B}(\mathrm{~g})$, as well as of the corresponding magnetic flutter components $(\mathrm{d}, \mathrm{f}, \mathrm{h})$, as a function of $\beta_{e}$ for trace impurity helium at $k_{y} \rho_{s}=0.3$. The open symbols indicate spectrum averaged values as described in the text.

FIG. 4. (Color online) Electromagnetic dependence of the trace impurity $E \times B$ and magnetic flutter (Fl) transport coefficients $D_{p} / \chi_{\text {eff }}(\mathrm{a}-\mathrm{d}), C_{T}$ (e-h) and $C_{P}(\mathrm{i}-\mathrm{l})$ for increasing impurity charge $Z_{p}$ with impurity mass $A_{p}=2 Z_{p}$. Left column plots show results for the ITG reference case, while right column plots show results for the TEM reference case.

FIG. 5. (Color online) Electromagnetic dependence of the trace impurity $E \times B$ and magnetic flutter transport coefficients $D_{p} / \chi_{\text {eff }}\left(\right.$ a-d), $C_{T}$ (e-h) and $C_{P}$ (i-l) for increasing impurity charge $Z_{p}$, while keeping the impurity mass fixed $\left(A_{p}=1\right)$. Left column plots show results for the ITG reference case, while right column plots show results for the TEM reference case. 
FIG. 6. (Color online) Electromagnetic dependence of $E \times B$ (left) and magnetic flutter (Fl, right) transport coefficients for the impurities tritium, helium, carbon, nickel and tungsten (at the ionization stage of $46^{+}$) using typical $\mathrm{H}$-mode profile data, as those predicted for the ITER standard scenario. The charge concentration is assumed to be $Z_{p} n_{p} / n_{e}=1 / 1000$.

FIG. 7. (Color online) Electromagnetic dependence of the ratio of the $E \times B$ (a) and magnetic flutter (b) off-diagonal contributions (including both thermodiffusion and pure convection) to the total diagonal diffusion, $R V / D_{p, t o t}$, for tritium, helium, carbon, nickel and tungsten (at a ionization stage of $46^{+}$), using typical $\mathrm{H}$-mode profile data as those predicted for ITER. The charge concentrations are assumed to be $Z_{p} n_{p} / n_{e}=1 / 1000$. The shaded areas denote the projected $\beta_{e}$ values for standard and hybrid operational scenarios. 

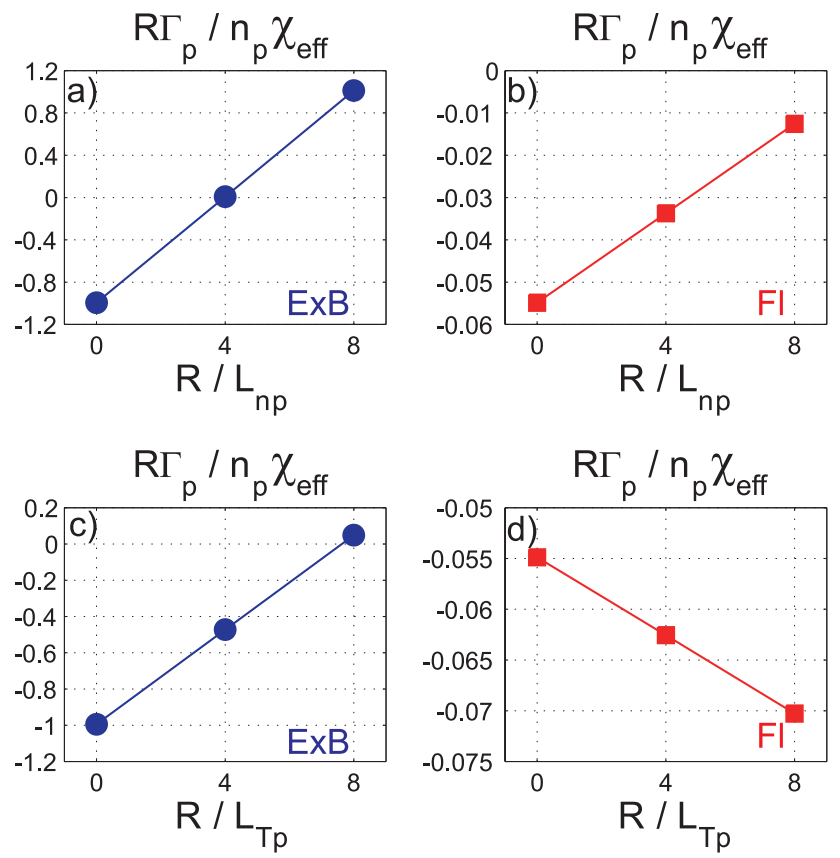

FIGURE 1. 

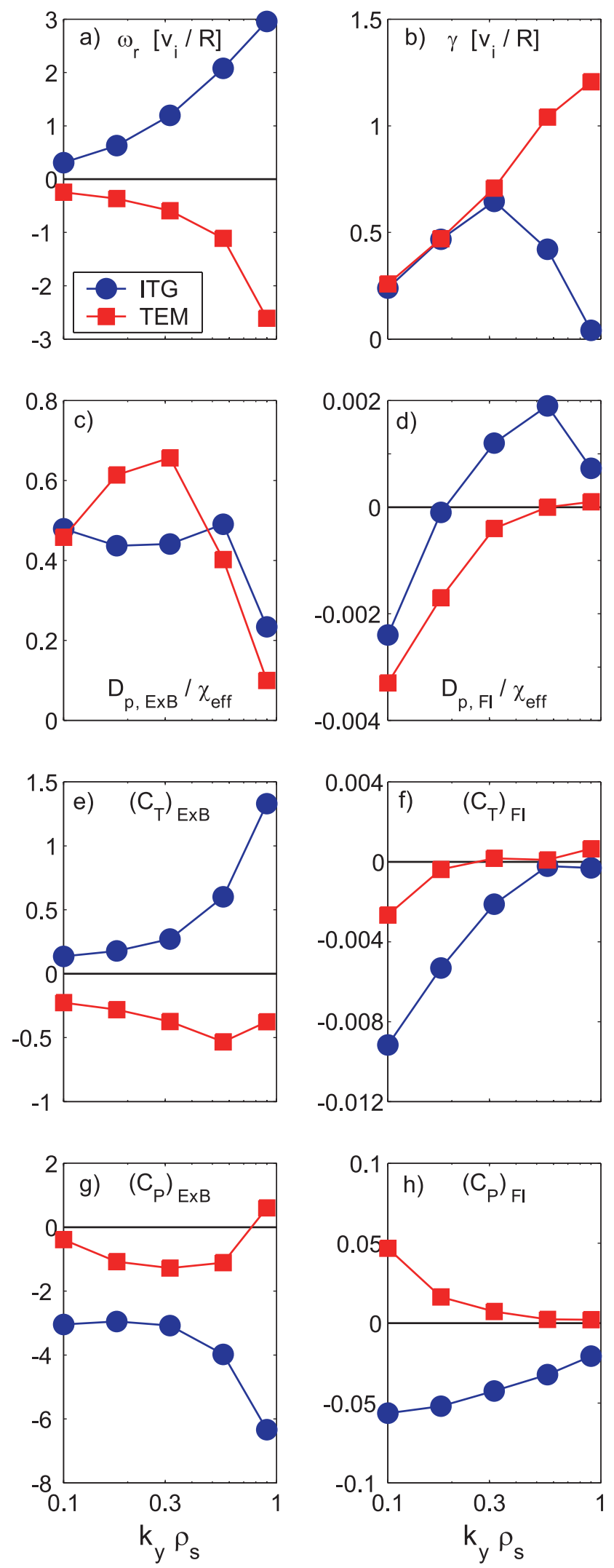

FIGURE 2. 

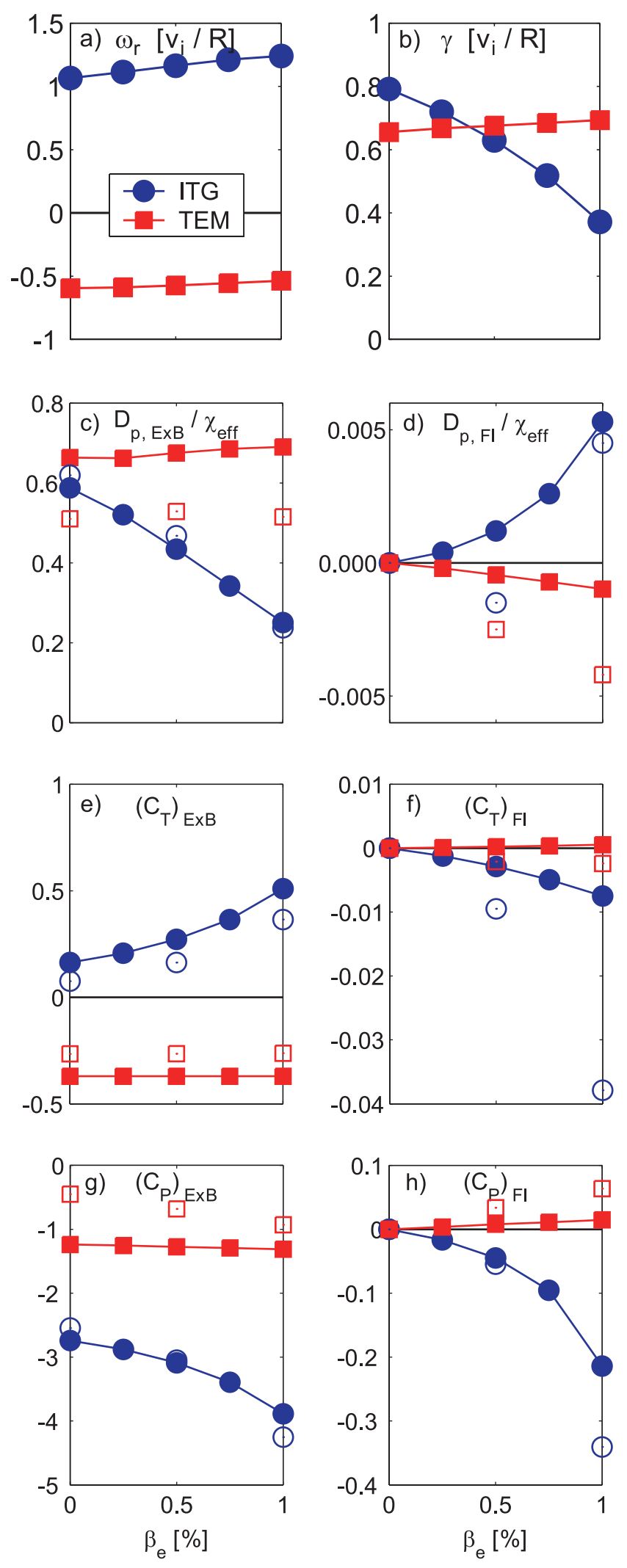

FIGURE 3. 


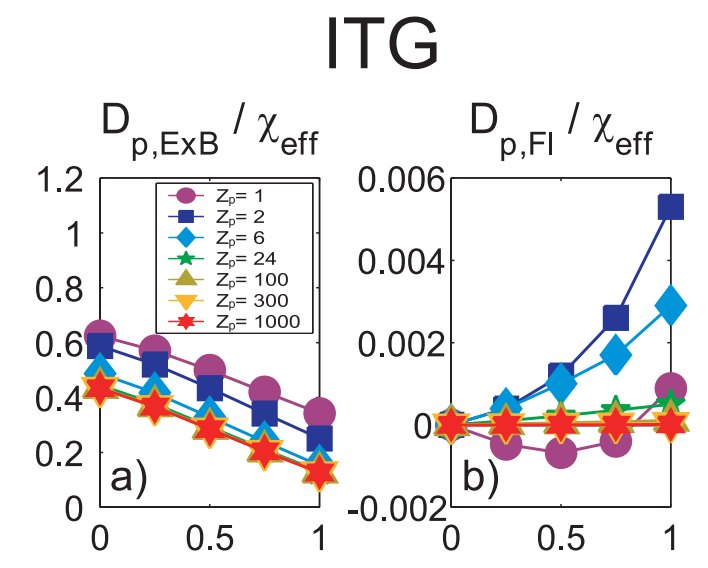

\section{TEM}
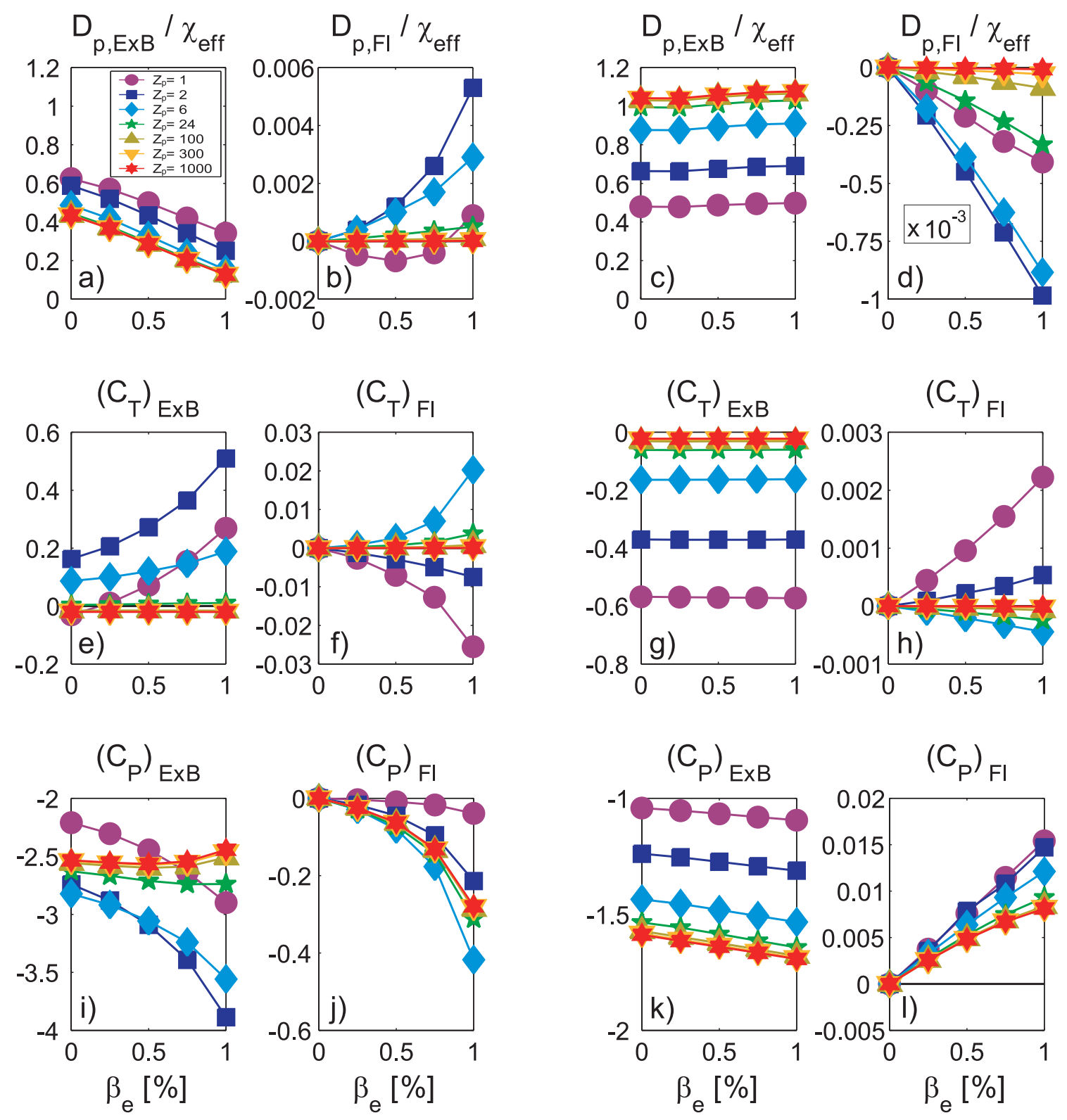

FIGURE 4. 
ITG
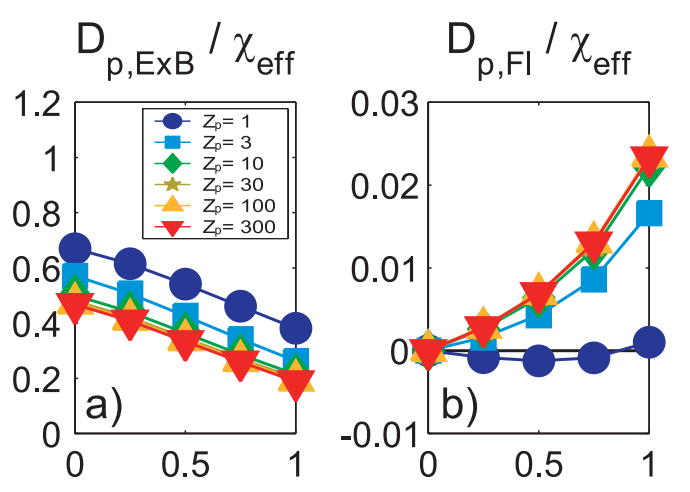

$\left(\mathrm{C}_{\mathrm{T}}\right)_{\mathrm{ExB}}$

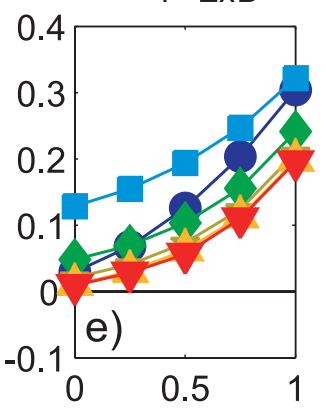

$\left(\mathrm{C}_{\mathrm{T}}\right)_{\mathrm{FI}}$

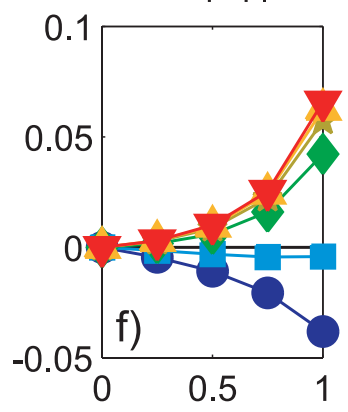

$\left(C_{P}\right)_{E x B} / Z_{p}$

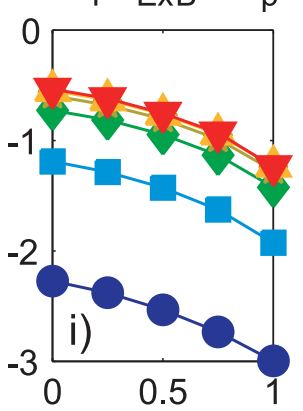

$\left(\mathrm{C}_{\mathrm{P}}\right)_{\mathrm{Fl}} / \mathrm{Z}_{\mathrm{p}}$

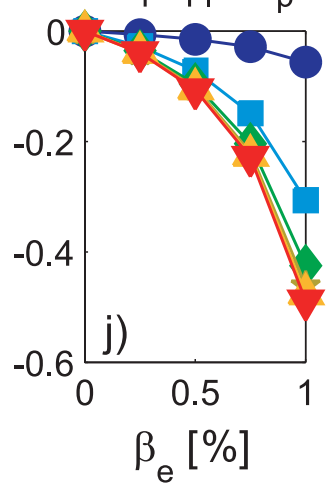

TEM

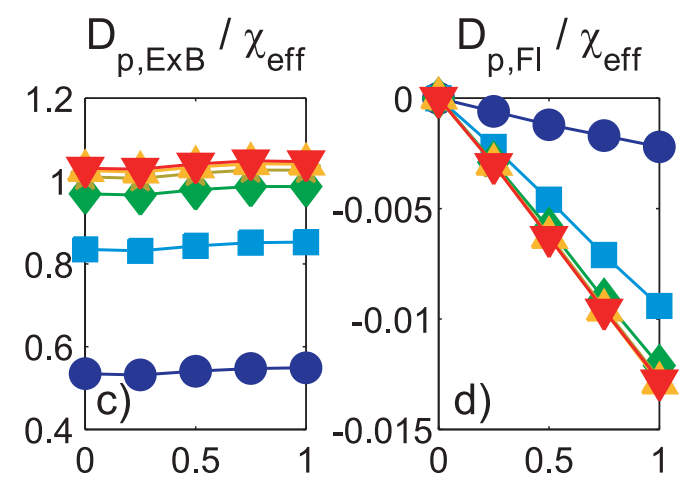

$\left(\mathrm{C}_{\mathrm{T}}\right)_{\mathrm{ExB}}$

$\left(\mathrm{C}_{\mathrm{T}}\right)_{\mathrm{FI}}$

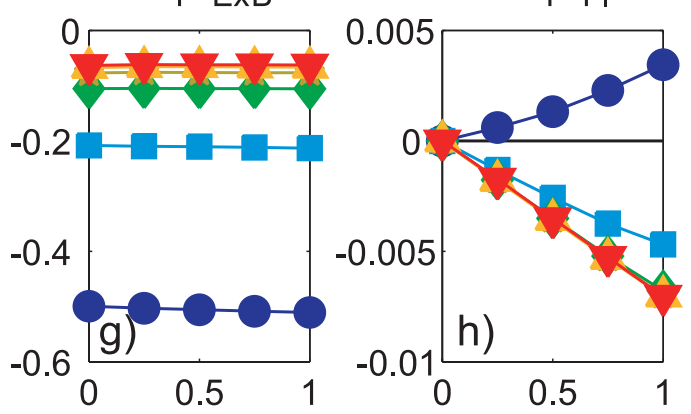

$\left(C_{\mathrm{P}}\right)_{\mathrm{ExB}} / \mathrm{Z}_{\mathrm{p}}$

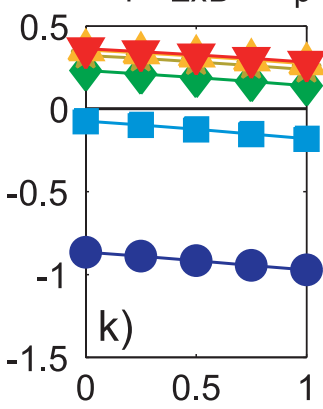

$\left(\mathrm{C}_{\mathrm{P}}\right)_{\mathrm{Fl}} / \mathrm{Z}_{\mathrm{p}}$

$\beta_{\mathrm{e}}[\%]$

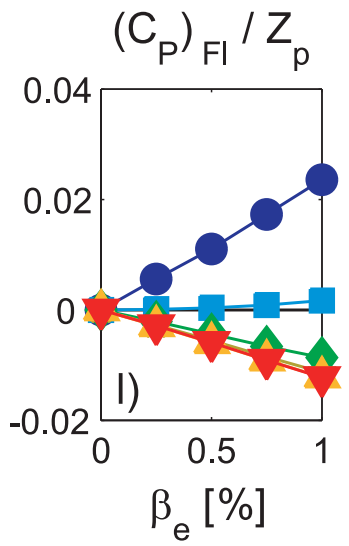

FIGURE 5. 

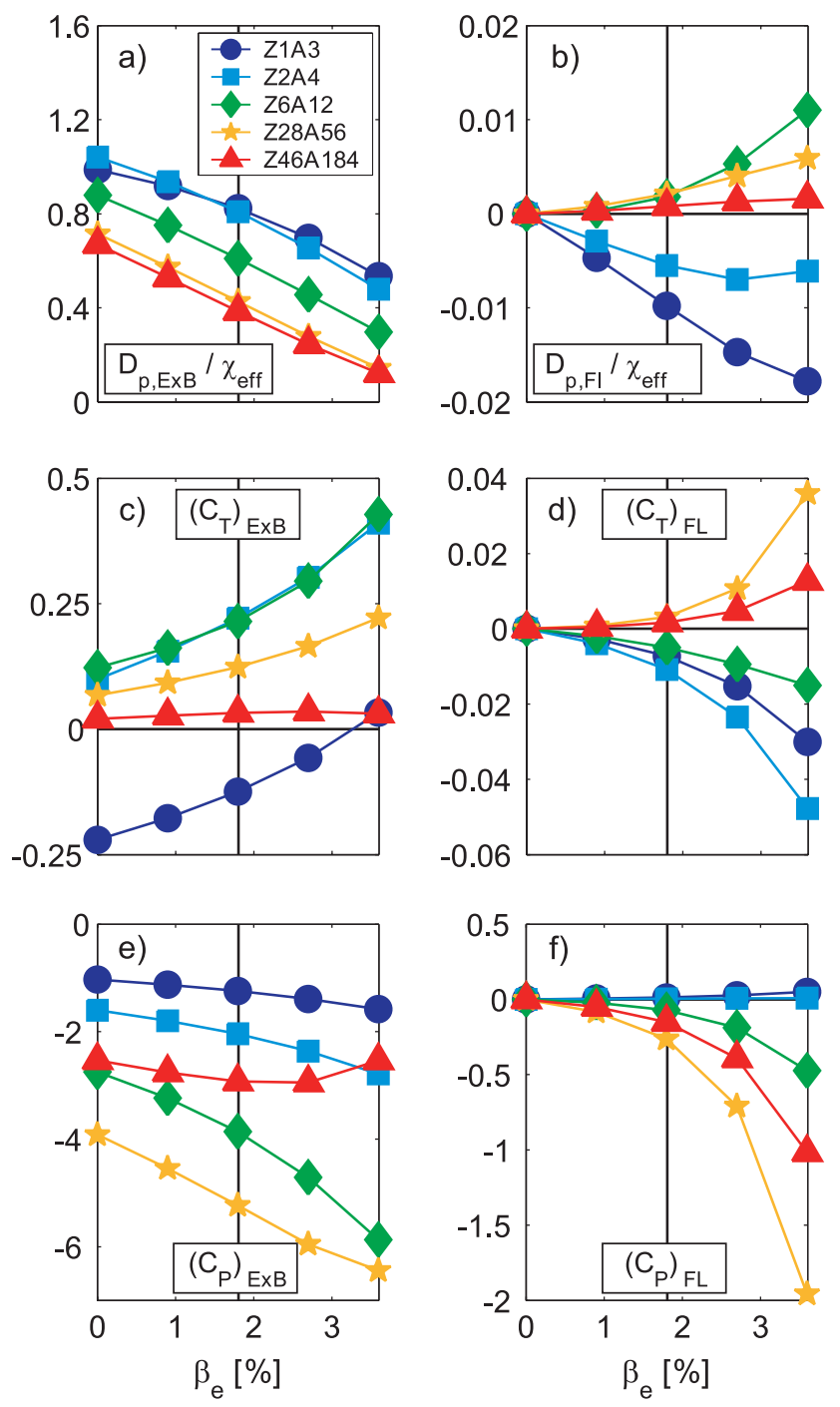

FIGURE 6.
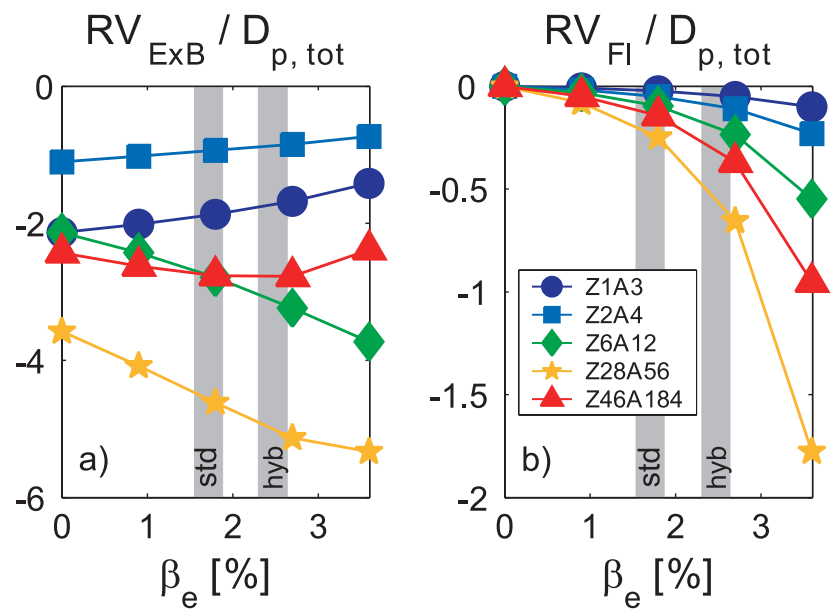

FIGURE 7. 